\title{
Optical Radiation Measurement
}

\author{
A. A. Gaertner \\ National Research Council of Canada, Ottawa \\ Canada
}

\section{Introduction}

Optical radiation bathes the world in which we live. The sun, moon and stars have provided us with our primary sources of light since before the beginning of life upon the earth. Very early in our history we observed that we could produce light by other means than depending upon the daily rotation of the earth. As our societies developed, in addition to creating intricate visual experiences with light, we discovered new ways of using this light to effect changes upon many of the materials we found in the world around us. As we sought to control these effects, we realised that the light itself had many properties that affected the outcome of our investigations and that we needed to untangle the characteristics of the light and the properties of the materials in response to this light. We discovered that there were effects caused by 'light' that we could not observe visually, which led to the distinction between light and radiation. To evaluate these effects, to discover new effects, and to produce (or reduce) them efficiently, we have come to depend critically upon the accurate measurement of the optical radiation required in our endeavors. In this chapter we will discuss some of the procedures and equipment necessary to obtain accurate measurements of the amount of optical radiation that we have available for our activities.

The measurement of optical radiation presents the practitioner with many opportunities and challenges. These stem from various sources: the properties of the radiation itself (wavelength, polarization), the geometrical configuration of the radiation field (direction, solid angle), the interaction of the radiation field with the detectors, devices and materials under test (responsivity, reflectance, transmittance), and, not in the least, to the vocabulary and definitions in use, locally and internationally, to describe the measurement process and the results of the measurements.

There are two international organizations whose work is particularly important for the fundamentals of measurement and of optical radiation measurement in particular:

1. BIPM, the Bureau International des Poids et Mesures, (www.bipm.org ): "The task of the BIPM is to ensure world-wide uniformity of measurements and their traceability to the International System of Units (SI)."

2. CIE, the Commission Internationale de L'Eclairage, (www.cie.co.at ): "The CIE is devoted to worldwide cooperation and the exchange of information on all matters relating to the science and art of light and lighting, colour and vision, photobiology and image technology." 


\section{Measurements, quantities, units and the SI}

As our measurements become more precise and more accurate, it becomes more and more important that we define precisely what we are measuring and what we mean by the terms that we are using to describe the measurements. We must also realize that the purpose of all measurements is to convey information-either to ourselves for some later use, or to someone else somewhere in the world. It is therefore critical that the meanings we associate with the terms we use are the same as the meanings used by the rest of the world. There are four documents that are of particular importance in optical radiation measurements:

1. the VIM (JCGM 200:2008), which provides a vocabulary for the basic concepts and terms of metrology,

2. the GUM (JCGM 100:2008), which provides a guide to the expression of uncertainty in measurement,

3. the SI brochure (BIPM, 2006), which provides a description of the International System of Units, and

4. the CIE Vocabulary (CIE S017, 2011), which provides the vocabulary for optical radiation, radiometry, photometry, lighting and colorimetry.

The concept of measurement involves several important aspects. The basic definition (VIM, Section 2.1) gives measurement as "process of experimentally obtaining one or more quantity values that can reasonably be attributed to a quantity". In addition to the 'process' of the measurement, some examples of which we will discuss in later sections of this chapter, there is the important requirement of a purpose or result to our process, and of a certain form to this result. We must consider a quantity and some means of attributing a value to this quantity. The definitions of quantity and quantity values are given (VIM, Sections 1.1 and 1.19) as:

A quantity is a "property of a phenomenon, body, or substance, where the property has a magnitude that can be expressed as a number and a reference". In this chapter we will discuss the measurement of several specific properties of optical radiation that determine the absolute amount of optical radiation that is available. In particular, we will consider the most important geometrical configurations for the amount of optical radiation (flux, intensity, irradiance, exitance, and radiance) and the spectral quantities associated with each of these geometrical quantities. We will also consider the quantities of optical radiation that are used to describe and measure the response of the human visual system to optical radiation (photometry, colorimetry). These latter quantities are the basis for the design and construction of lighting systems and facilities used for most human endeavors.

A quantity value is a "number and reference together expressing magnitude of a quantity".

In addition to stating that a quantity is something that must have a value, it states the form of a value as containing two parts: a number, and a reference. In all the examples we will be considering, a reference will be an entity called a measurement unit, which is defined (VIM, Section 1.9) as a "real scalar quantity, defined and adopted by convention, with which any other quantity of the same kind can be compared to express the ratio of the two quantities as a number". It can be seen from this definition that measurement units are the basis of our measurements. They are a quantity, just like the one that we are trying to measure ('same kind'), and they are the particular quantity that we use as the basis to obtain the magnitude 
of the quantity we are trying to measure. For example, the quantity of optical radiation flux is measured in terms of a specific amount of optical radiation flux-the watt.

Note that these definitions of quantity and measurement unit contain one of the key parts of a measurement process, which is to compare the two quantities, the unit and the quantity we wish to measure.

The 'defined and adopted by convention' aspect of the definition of measurement unit leads us to the SI (BIPM, 2006). This reference quantity ('real scalar quantity...of the same kind') could be a quantity of any size that is convenient for our immediate use. However, if the measurement is to convey any information to anyone else-our clients, an industrial factory, another scientist - or even to ourselves at a later time, we need to put qualifications on the properties of this reference quantity. As a minimum, we can see that it needs to be stable over time, and it needs to be accessible to any user of our data. The other users need to either have access to the same unit that we used, or they need to be able to create (realize) one of their own. For true universality, all measurements will need to be based upon reference quantities that have been agreed upon internationally. The present basis of international agreement on units of measurement is the Convention of the Metre (Convention du Mètre), which "is a treaty that created the International Bureau of Weights and Measures (BIPM), an intergovernmental organization under the authority of the General Conference on Weights and Measures (CGPM) and the supervision of the International Committee for Weights and Measures (CIPM)" (http://www.bipm.org/en/ convention/). The BIPM presently has fifty-five member states, including all the major industrialized countries. The present agreements upon the basic quantities and their units are contained in the SI brochure (BIPM, 2006). This agreement also contains information on the general consensus on how unit symbols and names, quantity symbols, and quantity values should be expressed.

While all these definitions may seem pedantic, they clarify many concepts that we often take for granted without realizing. The length of a piece of wood seems rather straightforward: we have the quantity length (the property of the piece of wood), with its magnitude as a number, such as 2, and a reference (measurement unit), such as the metre. In optical radiation we get into problems quickly because there are so many measurements, quantities and references (measurement units), that it is often difficult to sort out which of the words we are using applies to which of these concepts. The definitions in the VIM and in the SI help us to understand the framework of the measurement process. The definitions in the CIE vocabulary help us to standardize the terms and concepts in optical radiation measurements.

\section{Traceability chains and measurement uncertainties}

The SI definitions of the basic reference quantities and their units provide the most accurate basis for measurement that is presently available. Although the definitions of these units are constructed such that the units may be realized in any laboratory with the required skills and facilities, the realization of any of these units is not necessarily a simple matter. In general, the primary realization is performed in dedicated national facilities, called National Measurement Institutes (NMIs). At the NMI, this primary measurement standard is established using a measurement procedure that obtains the measurement result, not by 
comparison with a reference quantity of the same kind, but by a procedure where the reference is the definition of the measurement unit through its practical realization. In this manner, that primary measurement standard can be said to be "traceable to the SI", rather than to any other laboratory or NMI. For example, the SI unit for luminous intensity, the candela, is defined in terms of a specific amount of radiant intensity (Section 4.2.2). Calibration of sources based upon the realization of the candela is discussed in Section 7.0.

In all these measurements, the establishment of the reference and our comparison to the reference will be subject to some errors. It is not possible to establish a reference exactly to definition, as it is not possible to compare two quantities exactly. These errors may be systematic or random, leading to a measurement uncertainty, which is defined (VIM, Section 2.26) as a "non-negative parameter characterizing the dispersion of the quantity values being attributed to a measurand, based on the information used". This definition leaves it open as to the parameter used to describe the dispersion, as well as noting that the value depends upon the information used, implying that our information may not be complete and may include an element of belief rather than absolute knowledge (JCGM 104:2009). As a consequence, a measurement result becomes (VIM, Section 2.9) a "set of quantity values being attributed to a measurand together with any other available relevant information."

Since most measurements are carried out using references that are quantities of the same kind, and are not a primary measurement standard, these measurements and references must somehow be related to the primary measurement standard. This leads to a sequence of calibrations (calibration hierarchy, VIM Section 2.40) that leads from a reference to the final measuring system, where each calibration depends upon the outcome of the previous calibration. The metrological traceability chain is the sequence of measurement standards and calibrations that were used to relate the measurement result to the reference, and the metrological traceability is the property of the measurement result whereby the result can be related to the reference through a documented unbroken chain of calibrations, each contributing to the measurement uncertainty. An example of a calibration chain used at an NMI is discussed in Section 7.0.

\section{Optical radiation quantities and units}

Having set up this rather formal measurement structure, we can now consider in more detail the measurement of several basic optical radiation quantities used to determine absolute amounts of optical radiation. Human beings have been dealing with light ever since 'the beginning', so they have had a long time to build up a large vocabulary of terms. To understand these terms, we need to consider two important aspects of quantitative optical radiation measurements: the geometrical configuration of the optical radiation that we wish to measure, and the spectral components of this particular geometrical assembly of radiation. Optical radiation is a restricted wavelength range of the electromagnetic spectrum between X-rays and microwave radio waves, from approximately $1 \mathrm{~nm}$ to $1 \mathrm{~mm}$ (CIE S017, 2011). In this chapter, the focus of our discussions will be upon electromagnetic radiation with wavelengths from approximately $100 \mathrm{~nm}$ to $2500 \mathrm{~nm}$, which is an extension from the visible wavelength range, which is approximately from $360 \mathrm{~nm}$ to $830 \mathrm{~nm}$.

The basic quantity for the measurement of the amount of electromagnetic radiation is the energy of that radiation, measured with the SI reference unit the joule. This quantity is most 
useful when considering pulses or bursts of radiation that have a time limited duration. In this chapter we will deal with the continuous flow of radiation; hence the (energy per time) quantity flux, or power, will be the appropriate basic quantity for the measurement of the amount of radiation. The SI unit for the quantity flux is the watt (W).

The flux of interest will be the flux that is contained within certain geometrical constraints. There is a set of five geometrical configurations that can be used to form the basis of most radiation measurement quantities: total flux, intensity, radiance, exitance and irradiance. The first four of these quantities are characteristics of radiation emanating from a radiation source, whereas the fifth, irradiance, is characteristic of radiation incident upon a surface.

\subsection{Geometrical quantities and units}

\subsubsection{Solid angle}

The geometrical configurations of intensity and radiance make use of the 3-dimensional concept of a solid angle $(\Omega)$, which is analogous to the 2-dimensional plane angle $(\alpha)$. In Figure $1, l$ is the length of the arc of the circle subtended by the limits of the 2-D object at the center of the circle of radius $r$. The definition of the plane angle $\alpha$ is given by

$$
\alpha=\frac{l}{r}
$$

The SI unit for the plane angle is the radian (rad). A full circle is an angle of $2 \pi \mathrm{rad}$.
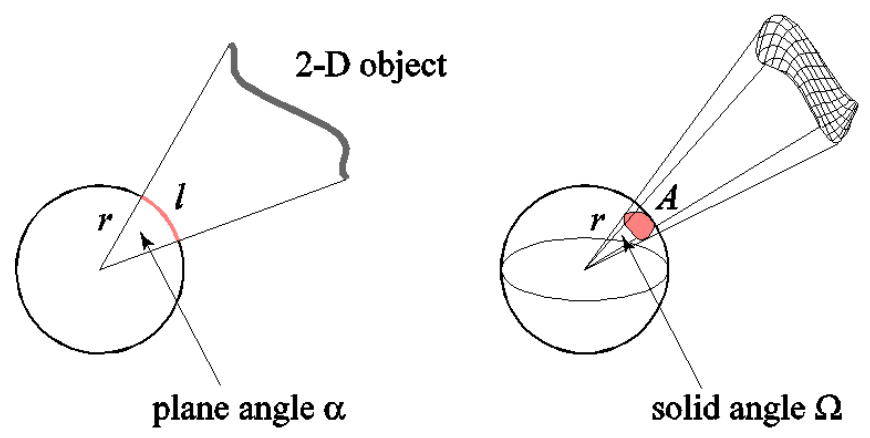

\section{3-D object}

Fig. 1. Two dimensional and three dimensional angles

Similarly, for the 3-D angle, called a solid angle, $A$ is the area of the part of the sphere of radius $r$ that is subtended by the limits of the 3-D object at the center of the sphere with radius $r$. The definition of the solid angle $\Omega$ is given by

$$
\Omega=\frac{A}{r^{2}}
$$

The SI unit for the solid angle is called the steradian (sr). The complete sphere is a solid angle of $4 \pi \mathrm{sr}$. 


\subsubsection{Total flux $(\Phi)$}

As indicated above, flux can be measured in many geometrical conditions. However, when radiation sources such as the common incandescent lamps or general lighting service lamps are measured, total flux is used to indicate the total flux output from the lamp into all directions. Specialized equipment, such as integrating spheres or goniophotometers, is required to collect the radiation output into all directions from the lamp (Ohno, 1997). The principles of operation using an integrating sphere are illustrated in Figure 2.

The inside surface of the integrating sphere is coated with a uniformly diffusing material (Lambertian Surface will be discussed in Section 4.1.7.2), such as PTFE (polytetrafluoroethylene) or $\mathrm{BaSO}_{4}$, to provide at the exit port a spatially homogeneous flux that is representative of the total flux output of the lamp. The purpose of the baffle is to prevent radiation directly from the lamp to be incident at the output port, before the sphere has had the opportunity to merge uniformly the radiation output from all directions from the lamp.

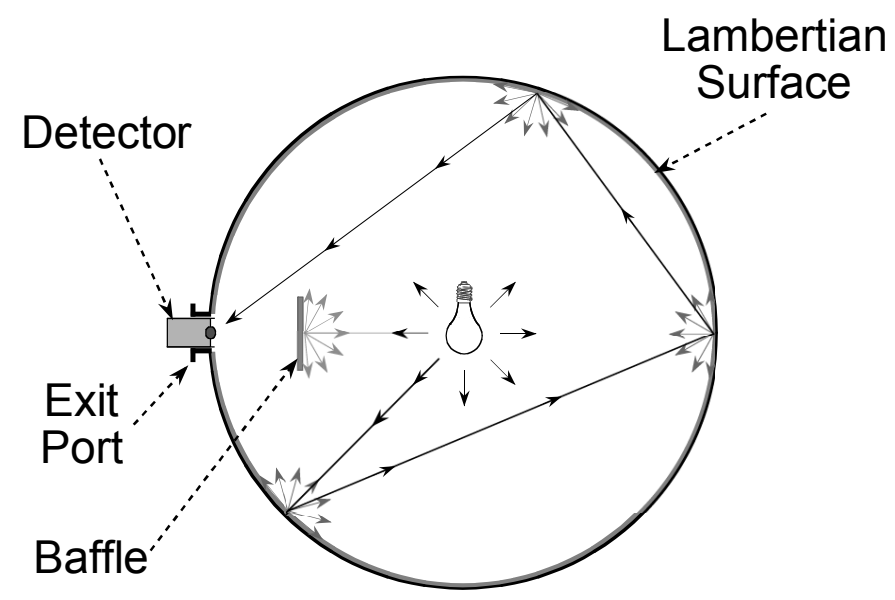

Fig. 2. Total flux measurement using an integrating sphere.

\subsubsection{Intensity $(I)$}

Intensity is used rather carelessly in daily use, even in physics. However, in optical radiation measurements it has a very specific meaning (Figure 3). First, the direction from the source $(\overrightarrow{\mathrm{d}})$ in which the intensity is to be defined must be indicated. Then the intensity of the source in this specified direction is defined as the ratio of the flux $(\Phi)$ leaving the source in that particular direction and propagating into an element of solid angle $(\Omega)$ containing that specified direction, divided by the size of that element of solid angle. The radiant intensity is defined as

$$
I=\frac{\Phi}{\Omega}
$$

with SI unit of watt per steradian $\left(\mathrm{W} \cdot \mathrm{sr}^{-1}\right)$. 


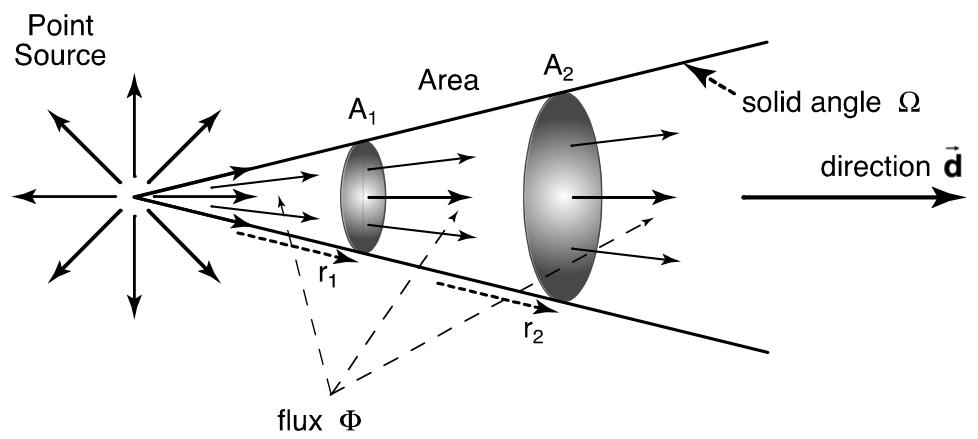

Fig. 3. Defining geometry for the quantity Intensity $(I)$ and origins of the inverse square law.

\subsubsection{Irradiance $(E)$}

The quantity irradiance is a measure of the amount of radiation incident upon a surface, as illustrated in Figure 4.

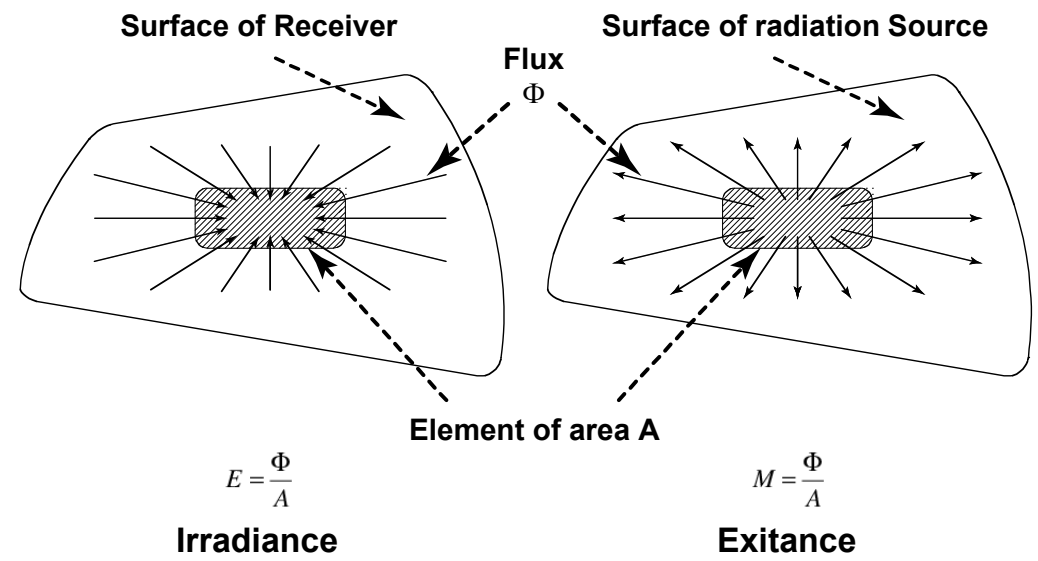

Fig. 4. Defining geometries for the quantities irradiance $(E)$ and exitance $(M)$.

The irradiance at the surface is defined as:

$$
E=\frac{\Phi}{A}
$$

where $\Phi$ is the total flux incident upon the surface element of area $A$. Irradiance is measured in terms of the SI unit of watt per square metre $\left(\mathrm{W} \cdot \mathrm{m}^{-2}\right)$.

\subsubsection{Exitance $(M)$}

Exitance is very similar to irradiance except that the direction of flow of the flux is reversed-it is leaving the surface, as shown in Figure 4. The radiant exitance from the surface is defined as: 


$$
M=\frac{\Phi}{A}
$$

where $\Phi$ is the total flux leaving the surface element of area $A$. Radiant exitance is measured in terms of the SI unit of watt per square metre $\left(\mathrm{W} \cdot \mathrm{m}^{-2}\right)$.

\subsubsection{Radiance $(L)$}

This quantity is the most detailed of the five geometrical quantities we are considering. It takes into account that a radiation source is not a point, but an extended surface, and that a radiation source does not emit the same flux into all directions. Therefore we have a quantity (Figure 5) that specifies the flux $(\Phi)$ emitted by a radiation surface from a specified area (A) on the surface and in a specified direction $(\overrightarrow{\mathbf{d}})$ from the surface, and into a specified solid angle $(\Omega)$ containing the given direction $\overrightarrow{\mathbf{d}}$. The vector direction $\overrightarrow{\mathbf{n}}$ is the perpendicular to the radiation source element of area A, and $\alpha$ is the angle between $\overrightarrow{\mathbf{n}}$ and $\overrightarrow{\mathbf{d}}$.

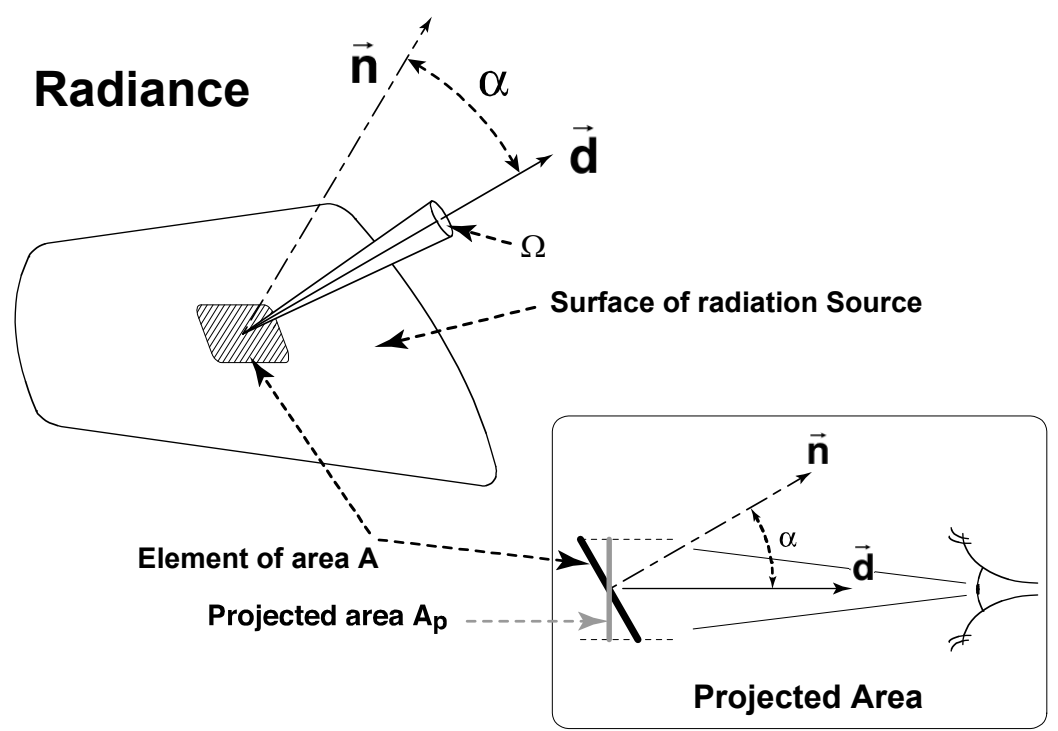

Fig. 5. Defining geometries for the quantity radiance and related projected area.

The radiance of the element of area is defined as

$$
L=\frac{\Phi}{A_{p} \cdot \Omega}
$$

The projected area $A_{p}$ is the size of the area $A$ as seen in the specified direction $\overrightarrow{\mathbf{d}}$ :

$$
A_{p}=A \cdot \cos \alpha
$$




\subsubsection{Quantity relationships}

Several useful relationships between the quantities discussed above may be obtained by adding specific geometric constructions and/or source constraints to the measurement configurations that use these quantities. These are particularly useful to change from a radiation source of one quantity to provide a radiation source of another quantity, such as using an irradiance source to provide a source of radiance.

\subsubsection{The inverse square law}

This is the most common and useful relationship. In its basic form, it relates the irradiance $(E)$ of a surface due to the output of a radiant intensity source $(I)$ placed at a known distance $(\mathrm{r})$ from the surface. The geometry is shown in Figure 3.

The same flux $\Phi$ passes through each of the areas $A_{1}$ and $A_{2}$. Since the sizes of these areas are increasing with distance $r$ from the source, it is evident that there will be less flux per unit area on surfaces farther from the source. From the definitions of intensity $(I)$, solid angle $(\Omega)$, and irradiance $(E)$, we may derive the relation between $E$, I, and $\mathrm{r}$ :

$$
\Phi=I \cdot \Omega=I \cdot \frac{A}{r^{2}}
$$

The irradiance on any of the surfaces is then obtained as

$$
E=\frac{\Phi}{A}=\frac{I}{r^{2}}
$$

This is a very useful concept, but some restrictions should be noted:

1. The source is a point source. A point source is a source that is so small that the inverse square law is valid. If the source is extended, the distance $r$ from the various points on the source to any area element that is being irradiated will change for all points on the source. The simple equations above are only valid for each point on the source to each point on the irradiated surface. For an extended source, the irradiance will need to be calculated as the sum from all points on the source. An example calculation (Grum \& Becherer, 1979) for the case of a circular source of uniform radiance shows the origins of the 'rule of thumb': if the distance $r$ is at least 10 times larger than the largest dimension of the source or detector, the error will be less than $1 \%$. However, if we depend on changing $r$ to adjust our required irradiance, good measurement practice would be to test the inverse square behaviour in our actual measurement conditions.

2. The radiation beam considered is not collimated, nor focused by lenses, nor a laser beam. It is the natural propagation of radiant energy from a point on the surface of a radiating source. Any modification of the radiation beam by lenses or the structure of the source will result in the radiant intensity of the source to change with distance from the source, or with the size of the solid angle, or with the direction of propagation with respect to the source. This will invalidate the application of the inverse square law to that particular source. As an example, the measurement of the radiation from LEDs is highly influenced by the geometrical structure in the output of these devices. As a result, the CIE has recommended very specific geometrical conditions for measuring 
the intensity of these devices (CIE 127, 2007). The recommended geometries prescribe exact solid angles and distances from the LED, together with specific considerations for the alignment of the LED with the measurement direction. Since the radiant intensity from LEDs is not constant with distance or solid angle, the resulting quantities measured are called Averaged LED Intensities, rather than true intensities as defined in Equation (3) above for a point source.

\subsubsection{Lambertian surface}

A Lambertian Surface is an ideal surface whose radiance is the same in all directions of the hemisphere above the surface. This very special surface has many useful properties. It is basically a radiation source that looks equally bright when viewed from any direction. It can be a radiation source itself, such as a black body radiator, or it can be a material that either transmits or reflects the radiation incident upon it in such a manner. Such a material is also called an isotropic diffusing surface. The basic concepts for such a reflecting diffusing surface are shown in Figure 6.

There are no real materials that have the ideal properties of a Lambertian surface, but pressed PTFE (polytetrafluoroethylene) powder or $\mathrm{BaSO}_{4}$ may be used if corrections for their non-ideality are made when required. The reflectance properties of these near-ideal diffusing reflectors are critical to accurate reflectance measurements and their threedimensional reflection properties are extensively studied (Höpe \& Hauer, 2010).

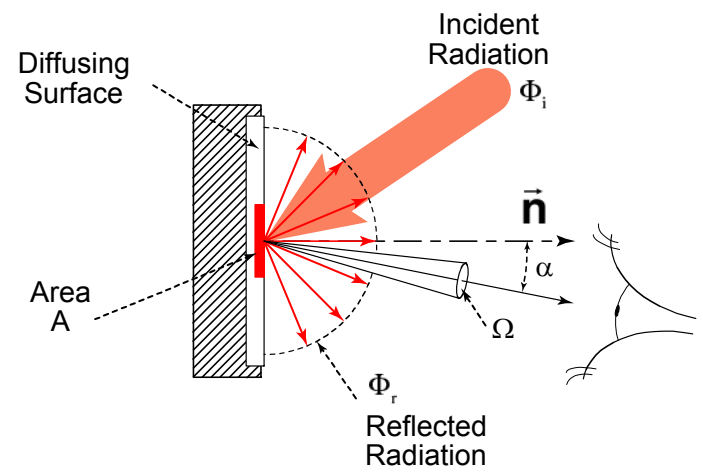

Fig. 6. An isotropic diffusing reflecting surface

\subsubsection{Irradiance to radiance}

The Lambertian diffusing reflecting surface is very useful in obtaining a radiance source from an irradiance source. When determining the relationship between radiometric quantities it is very useful to consider the flow of flux and then add the geometric constraints, as we did in Section 4.1.7.1 above. The geometric configuration shown in Figure 6 shows the conversion from the incident radiation from an irradiance source into the output reflected radiation with the properties of radiance. If the known irradiance at the area $A$ from our source is $E$, then the incident flux $\Phi_{i}$ upon the area $A$ is:

$$
\Phi_{i}=E \cdot A
$$


The flux reflected from the area $A$ is the incident flux times the reflectance $(\rho)$ of $A$ :

$$
\Phi_{r}=\rho \cdot \Phi_{i}=\rho \cdot E \cdot A
$$

We can relate the radiance $(L)$ of $A$ to the reflected flux $\Phi_{r}$ using the definition of radiance from Equation (6) and the assumption of a constant radiance in all directions (Lambertian surface):

$$
d \phi=L \cdot A \cdot \cos \alpha \cdot d \omega
$$

where $d \phi$ is a small element of flux emitted from $A$ into the small element of solid angle $d \omega$. The total flux emitted from $A$ by this constant radiance $L$ is obtained by integrating $d \phi$ over all the output directions from $A$ :

$$
\Phi_{r}=\int L \cdot A \cdot \cos \alpha \cdot d \omega=L \cdot A \cdot \int \cos \alpha \cdot d \omega=L \cdot A \cdot \pi
$$

Combining Equations (11) and (13) we obtain:

$$
L_{I D R}=\frac{\rho \cdot E}{\pi}
$$

Equation (14) is the basic equation relating the radiance of an isotropic reflecting diffuser due to a known irradiance of the diffuser, where we have introduced the subscript IDR to indicate that this is the radiance from an Isotropic Diffuse Reflector with reflectance $\rho$.

As indicated in the discussion of a Lambertian source, there is no perfect isotropic diffuser. This means that the reflectance $\rho$ in Equation (14) will not be the same for all directions of both the input irradiation and the output radiance. For accurate conversions from irradiance to radiance we will need to take account of this effect. We introduce a radiance factor $(\beta)$ that is defined (CIE S017, 2011) as: radiance factor (at a surface element of a non self-radiating medium, in a given direction, under specified conditions of irradiation): ratio of the radiance of the surface element in the given direction to that of the perfect reflecting or transmitting diffuser identically irradiated and viewed. The reflected radiance factor is denoted by $\beta_{R}$. For completeness in this description, we also show that the quantities we are discussing are functions of the wavelength $\lambda$, as well as angle.

For a perfect reflecting diffuser (PRD) illuminated by an irradiance $E(\lambda),(\rho=1)$, so that Equation (14) becomes:

$$
L_{P R D}(\lambda)=\frac{E(\lambda)}{\pi}
$$

Since this is a perfect reflecting diffuser, this equation is true for all directions of $L_{P R D}$ from the diffuser.

For an imperfect reflecting diffuser (RD) surface,

$$
\beta_{R}(\lambda, \vartheta)=\frac{L_{R D}(\lambda, \vartheta)}{L_{P R D}(\lambda, \vartheta)}
$$


where $\vartheta$ indicates the direction of the radiance $L$ from the surface, for some defined irradiation condition. From Equation (16) we obtain:

$$
L_{R D}(\lambda, \vartheta)=\beta_{R}(\lambda, \vartheta) \cdot L_{P R D}(\lambda, \vartheta)=\beta_{R}(\lambda, \vartheta) \cdot L_{P R D}(\lambda)
$$

with the second equality due to the fact that the PRD radiance $L_{P R D}(\lambda)$ is independent of angle.

Combining Equations (15) and (17) we obtain:

$$
L_{R D}(\lambda, \vartheta)=\beta_{R}(\lambda, \vartheta) \cdot L_{P R D}(\lambda)=\frac{\beta_{R}(\lambda, \vartheta) \cdot E(\lambda)}{\pi}
$$

In our application for converting from irradiance to radiance we usually have the irradiance incident normally upon the diffuser, and the radiance observed at $45^{\circ}$. Therefore, the specific reflectance factor we require is $\beta_{0 / 45}(\lambda)$, where the term $0 / 45$ indicates $0^{\circ}$ incidence radiation and $45^{\circ}$ output radiance. The final equation relating the input irradiance and the output radiance for this $0 / 45$ geometry is therefore:

$$
L_{R D}(\lambda, 45)=\frac{\beta_{0 / 45}(\lambda) \cdot E(\lambda)}{\pi}
$$

The radiance factor $\beta_{0 / 45}(\lambda)$ will need to be measured (Höpe \& Hauer, 2010) for the particular reflecting diffuser that we are using.

\subsubsection{Radiance to irradiance}

The transfer of radiant energy or flow of radiant flux from a radiating surface to a receiving surface is calculated in a general way for any geometrical configuration using elemental beams of radiance from small areas of the source propagating to small elements of area at the receiver (Grum \& Becherer, 1979). The simplest configuration that is often used in calibration laboratories is shown in Figure 7.

Source (radiance)

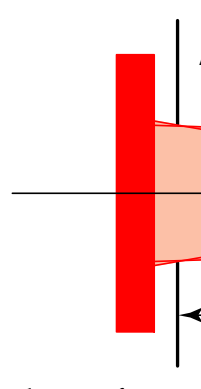

Receiver

Source Aperture $\mathrm{R}_{\mathrm{S}}$
Receiver Aperture $\mathrm{R}_{\mathrm{R}}$

Fig. 7. Irradiance from a Radiance Source

The source and receiver apertures are parallel and centered upon, and perpendicular to, the optical axis. The source is again assumed to be a Lambertian source with radiance $L$. The apertures are circular with radii $R_{S}$ and $R_{R}$ for the source and receiver respectively. Under 
these assumptions, the spatial fraction $f$ of the total flux emitted by the source radiance aperture that is collected within the receiver aperture is given (Walsh, 1958) by:

$$
f=2 R_{R}^{2}\left[\left(R_{S}^{2}+R_{R}^{2}+D^{2}\right)+\sqrt{\left(R_{S}^{2}+R_{R}^{2}+D^{2}\right)^{2}-4 R_{S}^{2} R_{R}^{2}}\right]^{-1}
$$

The radiant exitance of a Lambertian source is obtained by the integration of the constant radiance $L$ over the hemispherical solid angle to give (Grum \& Becherer, 1979):

$$
M=\pi \cdot L
$$

Therefore the total flux emitted by the source aperture is:

$$
\Phi_{S}=(\pi \cdot L) \cdot\left(\pi \cdot R_{S}^{2}\right)
$$

and the flux collected within the receiver aperture is:

$$
\Phi_{R}=f \cdot \Phi_{S}=\pi^{2} \cdot R_{S}^{2} \cdot f \cdot L
$$

The irradiance at the receiver aperture is therefore:

$$
E=\frac{\Phi_{R}}{\pi \cdot R_{R}^{2}}=\pi \cdot\left(\frac{R_{S}}{R_{R}}\right)^{2} \cdot f \cdot L
$$

As we will discuss in Section 7, Equation (23) is the basis for the calibration of blackbody sources, which are then used as absolute radiance sources for the realization of spectral irradiance scales using Equation (24).

\subsection{Spectral quantities and units}

The field of optical radiation measurements is often divided into three major categories: radiometry, photometry and colorimetry. Our discussion to this point has been primarily centered upon radiometry, the measurement of the quantities associated with optical radiation. However, human beings have been observing optical radiation for quite some time with their built-in detector - the eye. This has resulted in a very large and important field of radiation measurements. The difference between the three categories is basically that between the characterisation of the electromagnetic radiation that is present in our measurements (radiometry, which is essentially a detector that responds to all wavelengths equally), and the evaluation based on what human beings think is present using their visual detection system composed of the eye and the brain (photometry, colorimetry). Photometry evaluates the radiation as a measure of the strength or magnitude of the human visual response, whereas colorimetry characterizes optical radiation in terms of the human ability to distinguish radiation of different wavelengths, which we call colours.

\subsubsection{Spectral concepts}

The emission, reflection and absorption properties of the sources, detectors and other materials used for optical radiation measurement are all dependent upon the wavelength $\lambda$ 
of the radiation under consideration. All the geometrical quantities considered above should be considered to apply at each wavelength we use. The use of the adjective spectral, when applied to any of the quantities X, indicates (CIE S017, 2011) that either:

1. $X$ is a function of the wavelength $\lambda$, with symbol $X(\lambda)$, or

2. the quantity referred to is the spectral concentration, or spectral distribution, of $X$, with symbol $X_{\lambda}$. This spectral distribution $X_{\lambda}$ is defined as the quotient of the radiant quantity $\mathrm{d} X(\lambda)$ contained in an elementary range of $\mathrm{d} \lambda$ of wavelength at the wavelength $\lambda$, by that range:

$$
X_{\lambda}=\frac{\mathrm{d} X(\lambda)}{\mathrm{d} \lambda}
$$

It should be noted that $X_{\lambda}$ is also a function of wavelength $\lambda$, and to stress this fact, may be written as $X_{\lambda}(\lambda)$ without any change in meaning.

As examples, the quantities reflectance $(\rho)$ and radiance factor $(\beta)$ that we introduced above are functions of wavelength, but not spectral distributions of wavelength. The quantities that involve radiant flux are all spectral distributions. For example, spectral irradiance $\left(E_{\lambda}\right)$ is defined (CIE S017, 2011) as the quotient of the radiant power $d \Phi(\lambda)$ in a wavelength interval $\mathrm{d} \lambda$ incident on an element of a surface, by the area $\mathrm{d} A$ of that element and by the wavelength interval $\mathrm{d} \lambda$ :

$$
E_{\lambda}=\frac{\mathrm{d} E(\lambda)}{\mathrm{d} \lambda}=\frac{\mathrm{d}^{2} \Phi(\lambda)}{\mathrm{d} A \cdot \mathrm{d} \lambda}
$$

The basic concepts are shown in Figure 8. The SI unit for spectral irradiance is $W \mathrm{~m}^{-2} \mathrm{~m}^{-1}$, or $\mathrm{W} \cdot \mathrm{m}^{-3}$. The unit $\mathrm{W} \mathrm{m}^{-2} \mathrm{~nm}^{-1}$ is also used since the wavelength of optical radiation is conveniently measured with the unit $\mathrm{nm}$.

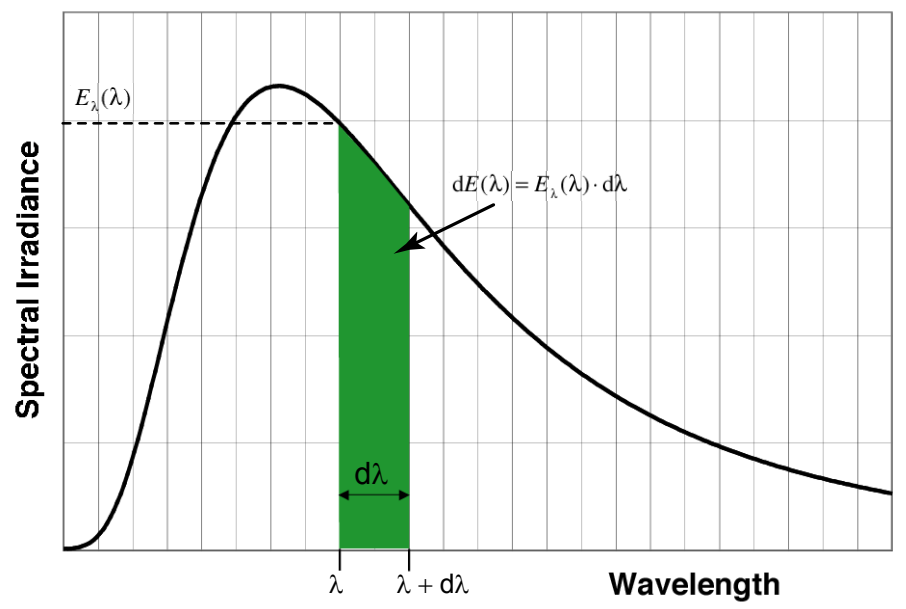

Fig. 8. Spectral distribution components for spectral irradiance 


\subsubsection{Photometry}

The retina of the human eye contains two different types of photoreceptors called rods and cones that produce nerve impulses that are passed on to subsequent stages of the human visual system for processing (Ohta \& Robertson, 2005). The cones are spread over the entire retina, together with a large concentration within a small central area of our vision called the fovea, which results our high visual acuity at the center of the field of view of the eye. The cones are responsible for our daytime colour vision. The rods are spread over the entire retina except the fovea and are responsible for our night-time, basically black-and-white, vision.

The eye is sensitive to radiation over a range of approximately 11 orders of magnitude from bright sunlight to a flash of light containing only a few photons. The change in size of the pupil area is only capable of controlling the radiation input to the retina by a factor of 12 . The remaining adaptation is provided by the rods and the cones. The high radiation range is mediated by the cones and is known as the photopic range. The low radiation range is mediated by the rods and is known as the scotopic range. The intermediate range is mediated by both the rods and the cones, and is known as the mesopic range.

Each human being will perceive differently the amount and colour of a given beam of radiation. In order to simplify calculations and to provide international standards for the measurement of quantities representing the strength of the human visual response to optical radiation, the CIE has standardized spectral weighting functions to be used for each of the three ranges of photometric measurements. The spectral weighting functions for the photopic and scotopic ranges (Figure 9) are known as the spectral luminous efficiency functions for photopic and scotopic vision, with symbols $V(\lambda)$ and $V^{\prime}(\lambda)$ respectively (CIE Standard S010/E:2004). The mesopic range requires a more complex weighting function based upon a gradual transition between $V(\lambda)$ and $V^{\prime}(\lambda)$ throughout the mesopic region that depends on the visual adaptation conditions (CIE 191:2010).

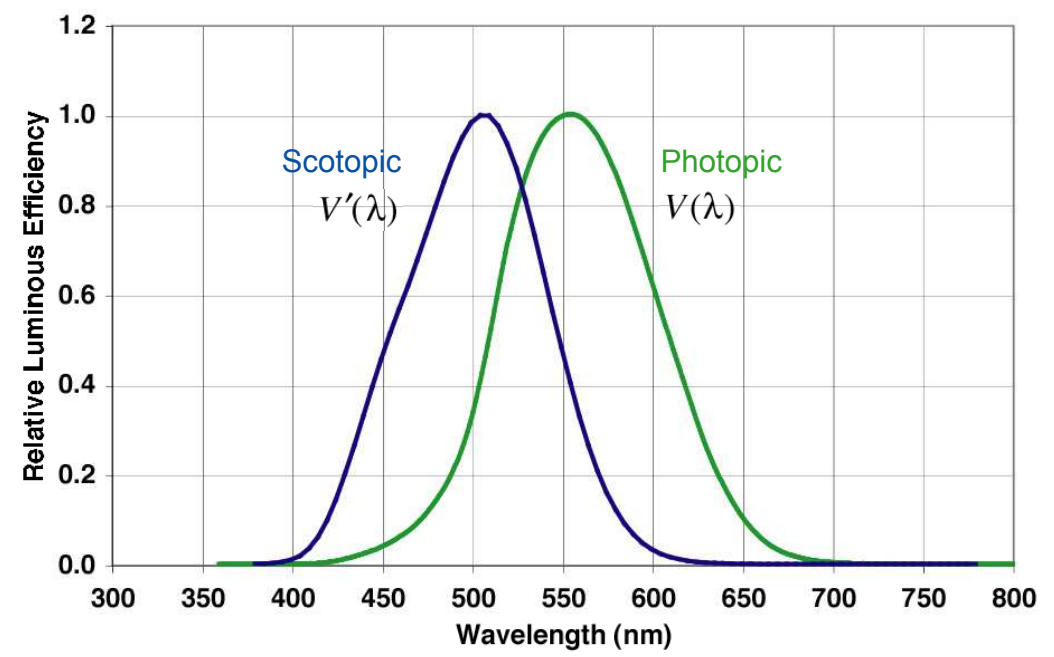

Fig. 9. CIE spectral luminous efficiency functions 
The CIE spectral luminous efficiency functions are relative spectral values with a maximum value of one. Within the SI, photometric quantities and units are obtained by calculation from radiometric quantities and units. This is facilitated with the definition of a quotient $\mathrm{K}$ called the luminous efficacy of radiation and defined as (CIE S017, 2011) the quotient of the luminous flux, $\Phi_{\mathrm{v}}$, by the corresponding radiant flux $\Phi_{\mathrm{e}}$. (The subscripts $\mathrm{v}$ and e are used to indicated photometric and radiometric quantities respectively.)

$$
K=\frac{\Phi_{\mathrm{v}}}{\Phi_{\mathrm{e}}}
$$

The spectral luminous efficiency functions provide the relative spectral values for $K(\lambda)$. In order to obtain the absolute scaling, there needs to be a defined scaling factor between radiometry and photometry at one of the wavelengths of the luminous efficiency functions. This is done in the definition (BIPM, 2006) of the SI base unit of luminous intensity, the candela:

"The candela is the luminous intensity, in a given direction, of a source that emits monochromatic radiation of frequency $540 \times 10^{12}$ hertz and that has a radiant intensity in that direction of $1 / 683$ watt per steradian. It follows that the spectral luminous efficacy for monochromatic radiation of frequency of $540 \times 10^{12}$ hertz is exactly 683 lumens per watt, $\mathrm{K}=$ $683 \mathrm{~lm} / \mathrm{W}=683 \mathrm{~cd} \mathrm{sr} / \mathrm{W} . "$

This value of $\mathrm{K}$ is the scaling factor for all the luminous efficiency functions. The value 683 was chosen to provide the best continuity between the size of the candela historically established using candles and platinum blackbodies before the change in 1979 to link the photometric units to the radiometric units. The definition is given for a specific frequency of radiation, which corresponds to approximately $555 \mathrm{~nm}$ in air (555.016 nm in standard air). The definition in terms of frequency avoids wavelength problems caused by the different index of refraction of different media of propagation. This does complicate slightly the determination of the maximum luminous efficacy value $K_{m}$ that is used to obtain photometric quantities from radiometric quantities using the CIE spectral luminous efficiency functions that are given as functions of wavelength (CIE S017, 2011). From these definitions we have (CIE S017, 2011): For photopic vision $K_{m}=683 V(555 \mathrm{~nm}) / V(555.016$ $\mathrm{nm}) \mathrm{lm} \cdot \mathrm{W}^{-1}=683.002 \mathrm{~lm} \cdot \mathrm{W}^{-1} \approx 683 \mathrm{~lm} \cdot \mathrm{W}^{-1}$. For scotopic vision $K_{m}^{\prime}=683 V^{\prime}(507 \mathrm{~nm}) /$ $V^{\prime}(555.016 \mathrm{~nm}) \quad \mathrm{lm} \cdot \mathrm{W}^{-1}=1700.05 \mathrm{~lm} \cdot \mathrm{W}^{-1} \approx 1700 \mathrm{~lm} \cdot \mathrm{W}^{-1}$. For all other wavelengths $K(\lambda)=K_{m} V(\lambda)$ and $K^{\prime}(\lambda)=K_{m}^{\prime} V^{\prime}(\lambda)$.

From these definitions, all the geometrical photometric quantities may be determined from the corresponding radiometric quantities discussed in Section 4.1 above. For example,

$$
\begin{aligned}
& \Phi_{\mathrm{v}}=K_{m} \int \Phi_{\mathrm{e}}(\lambda) \cdot V(\lambda) \cdot \mathrm{d} \lambda \\
& \Phi_{\mathrm{v}}^{\prime}=K_{m}^{\prime} \int \Phi_{\mathrm{e}}(\lambda) \cdot V^{\prime}(\lambda) \cdot \mathrm{d} \lambda
\end{aligned}
$$

where $\Phi_{\mathrm{v}}$ is the photopic luminous flux in units of lumens, $\Phi_{\mathrm{v}}^{\prime}$ is the scotopic luminous flux, also in units of lumens, $\Phi_{\mathrm{e}}(\lambda)$ is the spectral distribution of radiant flux in units of watts per nanometre and $\mathrm{d} \lambda$ is the wavelength in units of nanometre. 
The relationships between the radiometric and photometric quantities, units and symbols are shown in Table 1. Although luminous exitance has the same unit (lumen per square metre) as illuminance, the unit lux is reserved for illuminance only.

\begin{tabular}{|l|c|l|c|}
\hline \multicolumn{2}{|c|}{ Radiometry } & \multicolumn{2}{c|}{ Photometry } \\
\hline \multicolumn{1}{|c|}{ Quantity $($ symbol $)$} & Unit $($ symbol $)$ & Quantity $($ symbol) & Unit $($ symbol) \\
\hline Radiant Energy $\left(Q_{e}\right)$ & joule $(\mathrm{J})$ & Luminous Energy $\left(Q_{v}\right)$ & $\begin{array}{c}\text { lumen second } \\
(\mathrm{lm} \text { s })\end{array}$ \\
\hline Radiant Flux $\left(\Phi_{\mathrm{e}}\right)$ & watt $(\mathrm{W})$ & Luminous Flux $\left(\Phi_{\mathrm{v}}\right)$ & lumen $(\mathrm{lm})$ \\
\hline Radiant Intensity $\left(I_{e}\right)$ & $\begin{array}{c}\text { watt per steradian } \\
(\mathrm{W} / \mathrm{sr})\end{array}$ & Luminous Intensity $\left(I_{v}\right)$ & candela $(\mathrm{cd})$ \\
\hline Radiance $\left(L_{e}\right)$ & $\begin{array}{c}\text { watt per steradian per } \\
\text { square metre } \\
\left(\mathrm{W} /\left(\mathrm{sr} \mathrm{m}^{2}\right)\right)\end{array}$ & Luminance $\left(L_{v}\right)$ & $\begin{array}{c}\text { candela per square } \\
\text { metre }\left(\mathrm{cd} / \mathrm{m}^{2}\right)\end{array}$ \\
\hline Irradiance $\left(E_{e}\right)$ & $\begin{array}{c}\text { watt per square metre } \\
\left(\mathrm{W} / \mathrm{m}^{2}\right)\end{array}$ & Illuminance $\left(E_{v}\right)$ & $\begin{array}{c}\text { lumen per square } \\
\text { metre }\left(\mathrm{lm} / \mathrm{m}^{2}\right) \\
\text { lux }(\mathrm{lx})\end{array}$ \\
\hline Radiant Exitance $\left(M_{e}\right)$ & $\begin{array}{c}\text { watt per square metre } \\
\left(\mathrm{W} / \mathrm{m}^{2}\right)\end{array}$ & Luminous Exitance $\left(M_{v}\right)$ & $\begin{array}{c}\text { lumen per square } \\
\text { metre }\left(\mathrm{lm} / \mathrm{m}^{2}\right)\end{array}$ \\
\hline
\end{tabular}

Table 1. Radiometric and Photometric quantities, units and symbols.

\subsubsection{Colorimetry (Ohta \& Robertson, 2005)}

The ability of humans to distinguish radiation of different colours is due to the three types of cone cells that are present in the retina (Ohta \& Robertson, 2005). Based upon measurements of the human visual response, the CIE has standardized a system of colorimetry (CIE 015:2004) that enables color to be specified precisely for any arbitrary color stimulus. This $X Y Z$ color specification system is based upon the calculation of three colorimetric CIE tristimulus values $X, Y$ and $Z$ of a radiation source or colour stimulus $S(\lambda)$ as given in equation (29).

$$
\begin{aligned}
& X=K_{m} \int S(\lambda) \bar{x}(\lambda) \mathrm{d} \lambda \\
& Y=K_{m} \int S(\lambda) \bar{y}(\lambda) \mathrm{d} \lambda \\
& Z=K_{m} \int S(\lambda) \bar{z}(\lambda) \mathrm{d} \lambda
\end{aligned}
$$

The spectral shapes of the three CIE color matching functions $\bar{x}(\lambda), \bar{y}(\lambda)$ and $\bar{z}(\lambda)$ are shown in Figure 10. A virtual observer having these color matching functions is called the CIE 1931 Standard Colorimetric Observer or the CIE $2^{\circ}$ Colorimetric Observer, where the $2^{\circ}$ indicates that the color matching functions are based upon those measured for a visual $2^{\circ}$ field of view. Similar values are available for a $10^{\circ}$ field of view, called the CIE 1964 Standard Colorimetric Observer or the CIE $10^{\circ}$ Colorimetric Observer. 


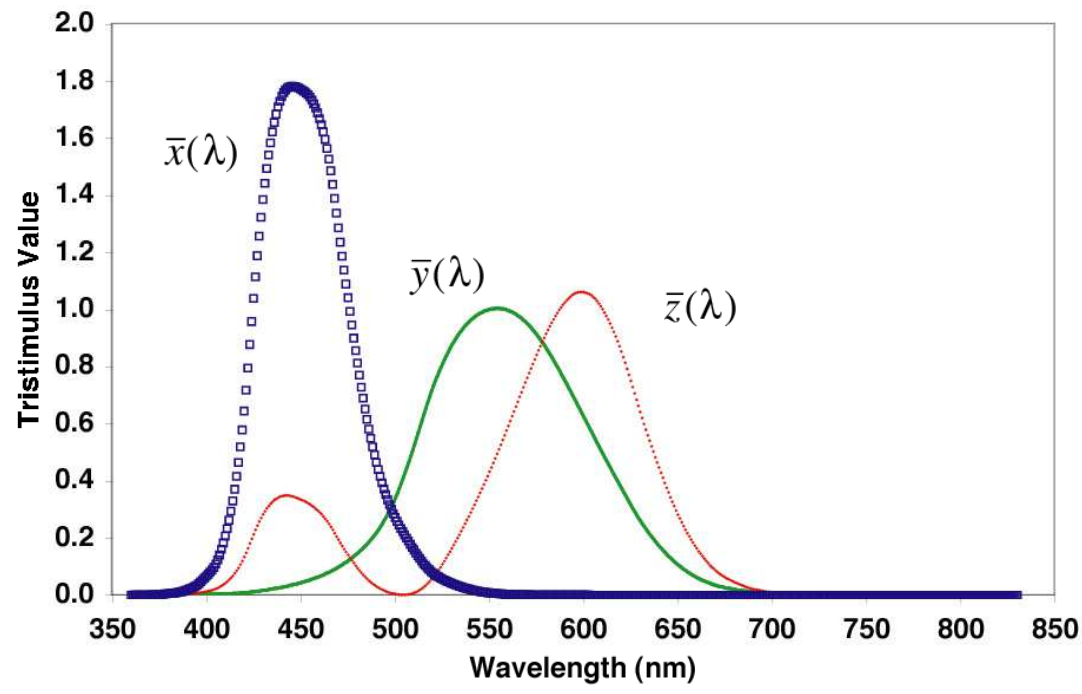

Fig. 10. CIE color matching functions

\subsubsection{Correlated Colour Temperature (CCT)}

Many of the radiation sources used as standards for radiometric and photometric measurements are incandescent lamps whose relative spectral output closely resembles that of a Planckian radiator or blackbody. Since the spectral radiation from a Planckian radiator can be described with the single temperature variable, it is often convenient to describe the colour of an incandescent source using only one variable similar to the absolute temperature used to describe the radiation from a Planckian radiator. For example, the electrical operating variables (current, voltage) of incandescent lamps used for many photometric standards and measurements are set such that the correlated colour temperature of the radiation from the lamp is approximately $2856 \mathrm{~K}$. This provides a spectral distribution that is reasonably reproducible for use in many photometric and colorimetric applications. The CIE Standard Illuminant A is a specified relative spectral distribution equal to that of a blackbody at a temperature of approximately $2856 \mathrm{~K}$ (CIE S 014-2, 2006)

The definition of correlated colour temperature (CCT) is standardized by the CIE (CIE S017, 2011) such that it may be calculated from the measured spectral radiation. The complete colour specification of a radiation source or color stimulus $S(\lambda)$ is three colorimetric tristimulus values as given in Section 4.2.3 above. In addition, the CIE defined the $\bar{y}(\lambda)$ colour matching function to be equal to the photometric $V(\lambda)$ spectral weighting function, so that the $Y$ tristimulus value holds all the information about the source brightness. This enabled the definition (Equation 30) of two normalized quantities $x$ and $y$, called chromaticity coordinates, that contain the colour information about the source.

$$
\begin{aligned}
& x=X /(X+Y+Z) \\
& y=Y /(X+Y+Z)
\end{aligned}
$$


This enables the representation of colours in two-dimensional plots. Note that for determination of the chromaticity coordinates, it is only necessary to know the relative spectral distribution for $S(\lambda)$. The chromaticity coordinates are shown in Figure 11 for several colour stimuli. The bounding curve for color stimuli is given by the spectrum locus, which is composed of the chromaticity coordinates of all the pure monochromatic wavelengths of radiation, and the purple boundary, which is the line joining the ends of the spectrum locus. The chromaticity coordinates of blackbody radiators form a smooth curve within the spectrum locus. This smooth curve gives us the basis for our single variable color temperature definitions. If the source has a relative spectral distribution equal to a Planckian radiator, its chromaticity coordinates will fall upon this curve and the source is said to have a color temperature $T_{c}$ equal to that of the corresponding blackbody radiator with the same chromaticity coordinates. The radiation from most practical sources will have a chromaticity that does not equal that of a blackbody. In this case their correlated colour temperature $T_{c p}$ is defined as the temperature of the blackbody whose chromaticity is nearest to that of the radiation. There are several methods of determining $T_{c p}$ from the relative spectral distribution of the stimulus $S(\lambda)$ (Ohta \& Robertson, 2005, Gardner, 2000).

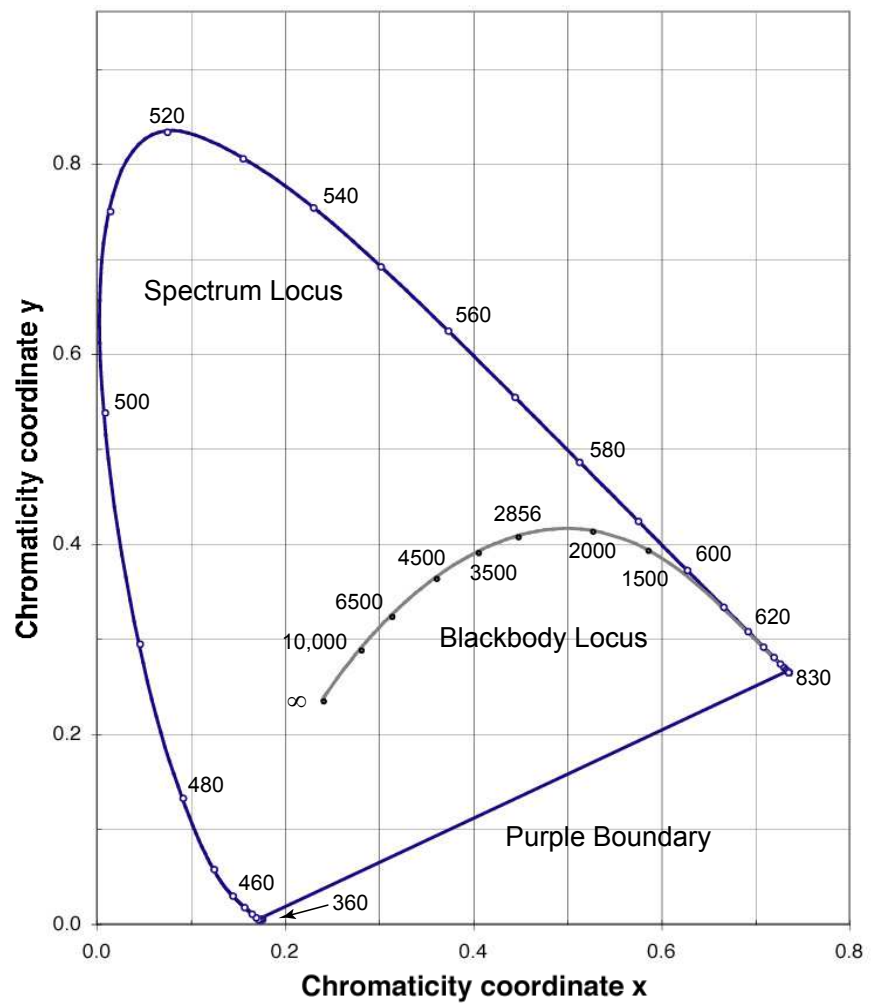

Fig. 11. CIE xy chromaticity diagram of the XYZ color specification system. The points indicated along the Spectrum Locus are monochromatic wavelengths in units of nanometres. The points indicated along the Blackbody Locus are temperatures of Planckian radiators in units of Kelvin. 


\subsubsection{Spectral mismatch correction factor $\left(F^{*}\right)$}

The definitions of photometric and colorimetric quantities given above require knowledge of the spectral distribution of the radiation that we are measuring. Most of the instruments in daily use for photometric and colorimetric measurements are simple devices that do not contain the spectroradiometers necessary for the measurement of spectral distributions. The simplest photometers and colorimeters are constructed from detectors, usually of silicon, and transmitting color glass filters. The types and thicknesses of the 3-to-4 types of glasses are chosen such that the relative spectral responsivity of the combination of the detector plus filter is as close to the desired photometric or colorimetric functions (e.g. $V(\lambda), V^{\prime}(\lambda)$, $\bar{x}(\lambda), \bar{y}(\lambda)$ or $\bar{z}(\lambda))$ as possible for the construction cost involved. These devices produce a single-number result for the measurement. Since the output of a detector is a voltage or current, this value must be converted to the corresponding photometric or colorimetric quantity by a calibration of the device, which is usually performed by a measurement of a known amount of the desired quantity. This produces a calibration factor that is often built into the electronics of the device.

These CIE spectral functions are not easy to reproduce in this physical form and there will always be errors or uncertainties in measurement using these devices. An estimate of the error involved can be determined by calculating what is called a Spectral Mismatch Correction Factor $\left(F^{*}\right)$. To derive this factor we compare the quantity that we wish to measure with the quantity that we are actually measuring, using illuminance as an example as shown in Figure 12. The photometer in this case is called an illuminance meter or luxmeter.

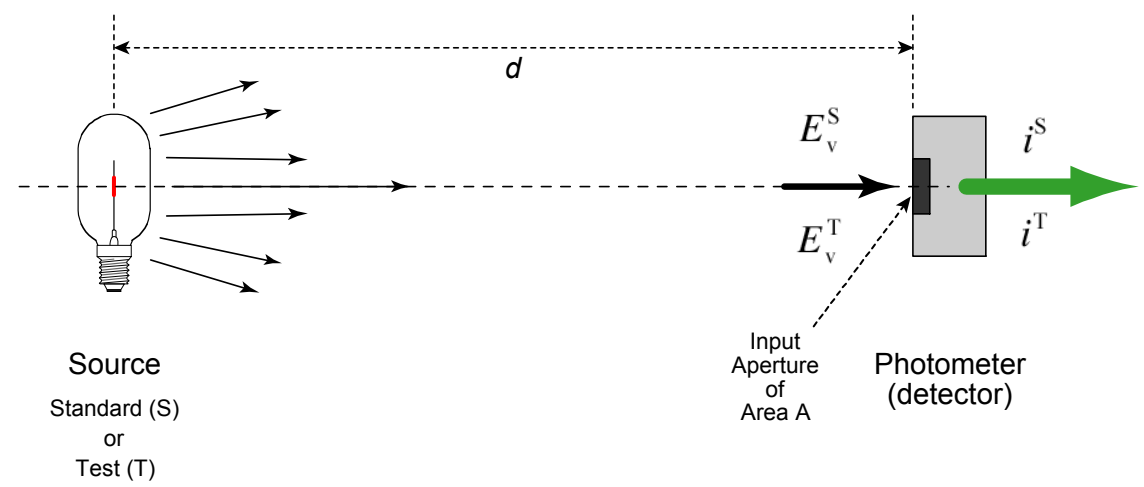

Fig. 12. Basic measurement and calibration configuration

The variables that describe the measurement shown in Figure 12 are:

Required value: $E_{\mathrm{v}}^{\mathrm{T}}$, the illuminance produced by the test source.

Known value: $E_{\mathrm{v}}^{\mathrm{S}}$, the illuminance produced by the standard source.

Measured values: $\quad i^{\mathrm{S}}$, the photometer output for the standard source. $i^{\mathrm{T}}$, the photometer output for the test source.

Note that the output of the photometer is a single number. 
We use the material properties of the source output and the photometer components to determine the origin of our measured photometer outputs. The variables are:

$P_{\mathrm{e}}^{\mathrm{T}}(\lambda)$, the relative spectral irradiance produced by the test source,

$P_{\mathrm{e}}^{\mathrm{S}}(\lambda)$, the relative spectral irradiance produced by the standard source, and

$R(\lambda)$, the relative spectral responsivity of the photometer.

The geometrical factors that convert these relative values to absolute values may be written as $a, b$ and $r$ respectively. The photometer outputs for the two sources can then be written:

$$
\begin{aligned}
& i^{\mathrm{T}}=a \cdot r \cdot \int_{\text {all wavelengths }} P_{\mathrm{e}}^{\mathrm{T}}(\lambda) \cdot R(\lambda) \cdot \mathrm{d} \lambda \\
& i^{\mathrm{S}}=b \cdot r \cdot \int_{\text {all wavelengths }} P_{\mathrm{e}}^{\mathrm{S}}(\lambda) \cdot R(\lambda) \cdot \mathrm{d} \lambda
\end{aligned}
$$

The measurements we require are the two illuminances:

$$
\begin{aligned}
& E_{\mathrm{v}}^{\mathrm{T}}=a \cdot v \cdot \int_{360 \mathrm{~nm}}^{830 \mathrm{~nm}} P_{\mathrm{e}}^{\mathrm{T}}(\lambda) \cdot V(\lambda) \cdot \mathrm{d} \lambda \\
& E_{\mathrm{v}}^{\mathrm{S}}=b \cdot v \cdot \int_{360 \mathrm{~nm}}^{830 \mathrm{~nm}} P_{\mathrm{e}}^{\mathrm{S}}(\lambda) \cdot V(\lambda) \cdot \mathrm{d} \lambda
\end{aligned}
$$

Note that $\mathrm{V}(\lambda)$ is exactly zero outside the $360-830 \mathrm{~nm}$ range, whereas the sources and the photometer might very well be contributing a signal outside this range. By taking ratios of the equations we may eliminate the geometrical constants and scaling factors $a, b, \mathrm{r}$ and $v$ to obtain the desired illuminance of the test source:

$$
\begin{aligned}
E_{\mathrm{v}}^{\mathrm{T}} & =\frac{E_{\mathrm{v}}^{\mathrm{S}}}{i^{\mathrm{S}}} \cdot i^{\mathrm{T}} \cdot \frac{\int_{360 \mathrm{~nm}}^{830 \mathrm{~nm}} P_{\mathrm{e}}^{\mathrm{T}}(\lambda) \cdot V(\lambda) \cdot \mathrm{d} \lambda}{\int_{\text {all wavelengths }} P_{\mathrm{e}}^{\mathrm{T}}(\lambda) \cdot R(\lambda) \cdot \mathrm{d} \lambda} \cdot \frac{\int_{\text {all wavelengths }} P_{\mathrm{e}}^{\mathrm{S}}(\lambda) \cdot R(\lambda) \cdot \mathrm{d} \lambda}{\int_{360 \mathrm{~nm}}^{830 \mathrm{~nm}} P_{\mathrm{e}}^{\mathrm{S}}(\lambda) \cdot V(\lambda) \cdot \mathrm{d} \lambda} \\
& =\mathrm{CF} \cdot i^{\mathrm{T}} \cdot F^{*}
\end{aligned}
$$

where $\mathrm{CF}$ is a photometer Calibration Factor that is stored in the electronics of the photometer when the photometer is calibrated with the standard source. This CF converts the measured $i^{\mathrm{T}}$ into $E_{\mathrm{v}}^{\mathrm{T}}$ when the test source is measured. However, the $F^{*}$ term is not included in the photometer measurement or calibration. It can only be determined at the point of measuring the test source, since $P_{\mathrm{e}}^{\mathrm{T}}(\lambda)$ is required. In general, $P_{\mathrm{e}}^{\mathrm{T}}(\lambda), P_{\mathrm{e}}^{\mathrm{S}}(\lambda)$ and $R(\lambda)$ are not known for most photometers and measurements. A standard source that approximates CIE Source A is usually used for the calibration of photometers. The CIE functions are defined, and generic values for $P_{\mathrm{e}}^{\mathrm{T}}(\lambda), P_{\mathrm{e}}^{\mathrm{S}}(\lambda)$ and $R(\lambda)$ may be used to obtain an estimate of the $F^{*}$ for the photometer or colorimeter and radiation test source in use. The $F^{*}$ can approach 1.0 under one or both of two conditions: 1) if the relative spectral distributions of the standard source and the test source are the same or proportional, i.e. $P_{\mathrm{e}}^{\mathrm{T}}(\lambda) \propto P_{\mathrm{e}}^{\mathrm{S}}(\lambda)$. This equivalence is one of the bases for the incentive in measurement or calibration to compare 'like-with-like'. 2) if the relative spectral responsivity of the photometer is proportional to the CIE function $(\mathrm{V}(\lambda)$ in our example above). This requires some effort on the part of manufacturers and photometers/colorimeters of many different qualities and cost are available. The evaluation of the quality of photometers and colorimeters has been documented by the CIE in various publications (CIE 179:2007, CIE 069:1987). 


\section{Measurements and calibrations}

In Section 2 we noted that one of the key parts of a measurement process was to compare two quantities, the quantity we wish to measure and the quantity unit. For most measurements the quantity unit is embodied in a calibrated measurement standard whose quantity value is a multiple or submultiple of the base unit. For example, a standard luminous intensity lamp may produce a luminous intensity of $219.2 \mathrm{~cd}$. To measure the luminous intensity output of another (test) light source, we will need some means of comparing the output luminous intensity of the test source with that of the standard source. This involves the introduction of a third device, that of a transfer device. In our example, this device must be able to compare the luminous intensity of both sources and return values for each lamp that are proportional to the luminous intensity of the lamp, or a value that is the ratio of the luminous intensities of the two sources.

For accurate measurements, it is imperative that the transfer device be capable of comparing the exact quantity that we wish to measure. This includes both the geometrical aspects and the spectral aspects that we discussed in Section 4 . The general schematic for this process is shown in Figure 13.

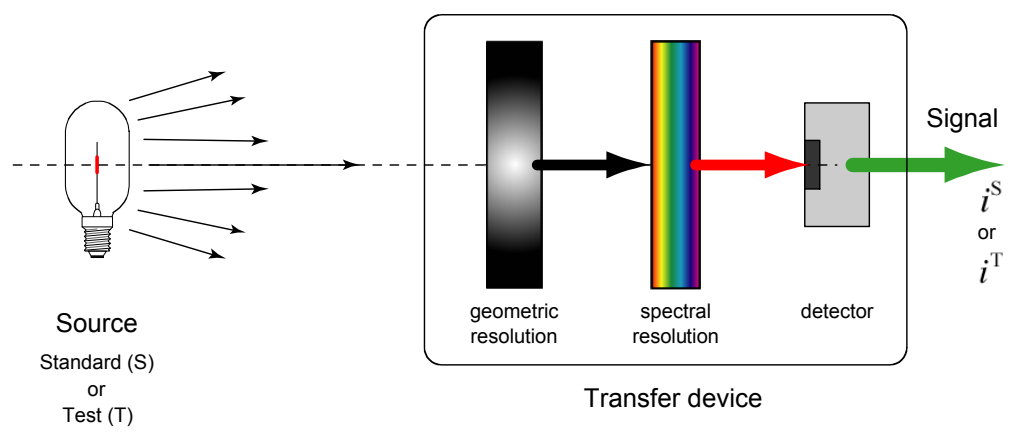

Fig. 13. Measurement Transfer Device

The spectral resolution component indicated for the transfer device is not necessarily the only spectrally important component. In the case of photometric and colorimetric measurements the spectral responsivity of the complete transfer device must be equal to the desired CIE function. If this is not the case, an $F^{*}$ will need to be determined as indicated in Section 4.2 .5 above.

If the transfer device is only used to measure the output of the test source by comparison with the standard source, the measured value of the test source is simply:

$$
S^{\mathrm{T}}=\frac{i^{\mathrm{T}}}{i^{\mathrm{S}}} \cdot S^{\mathrm{S}}=\frac{S^{\mathrm{S}}}{i^{\mathrm{S}}} \cdot i^{\mathrm{T}}
$$

where $S^{\mathrm{T}}$ and $S^{S}$ are the desired quantities for the test source and the standard source, and $i^{\mathrm{T}}$ and $i^{\mathrm{S}}$ are the signals from the transfer device when measuring these sources. An $F^{*}$ is to be applied to Equation (34) as necessary. If the standard source is a measurement 
standard with accompanying uncertainties, and the uncertainties of the transfer process are accounted for, the test source is now said to be calibrated. (VIM, Section 2.39).

If the intent is to use the transfer device for subsequent measurements of the same quantity, the factor $\frac{S^{S}}{i^{S}}$ may be considered a calibration factor for the transfer device, to be used to convert the subsequent $i^{\mathrm{T}}$ measurements into the corresponding $S^{\mathrm{T}}$. If the uncertainties of the standard and the process of measurement of the standard by the transfer device are considered and included, the transfer device may now be said to be calibrated for the measurement of the quantity of the standard. The calibration factor will have units of the ratio of the units of the standard source to the units of the signal. In the case of an illuminance meter calibration using the configuration of Figure 12, the units of the calibration factor for the photometer would be lux per ampere.

Figure 13 shows the transfer device as a detector unit. It is also possible that a radiation source be a transfer device. For example, if we wish to transfer a calibration between two photometers, from a calibrated standard photometer to a test photometer, we would use an incandescent lamp as the transfer device. Although it would not be necessary that this lamp be calibrated, the lamp should produce a geometric and spectral radiation field that is appropriate for the measurements to be performed by the photometers and the quantity calibration to be transferred between the photometers.

There are many measurement configurations possible that depend upon the type of measurement standard that is available and the required measurement. We will discuss several of the common configurations in the following subsections.

\subsection{Total flux}

The measurement transfer device for total flux is the integrating sphere and detector unit shown in Figure 2, where the detector could be a spectroradiometer for spectral radiant flux measurements. The standard source and the test source are sequentially placed at the center of the sphere and the signal from their flux output is recorded at the detector. The measured/calibrated total flux for the test source is given by Equation (34), with an $F^{*}$ applied for photometric measurements. In addition to the detector, the integrating sphere walls and all the interior components such as baffles and lamp supports must be considered as part of the responsivity $R(\lambda)$ of this transfer device. If the standard source and the test source have different output spatial distributions, any geometrical differences in the responsivity of the sphere may need to be considered. A detailed consideration of integrating sphere measurements has been given by (Ohno, 1997).

\subsection{Intensity and irradiance/illuminance}

These two geometric quantities are often considered together since the measurement configuration, shown in Figure 12, is the same for both. The analysis of the measurement equations is based upon the observation that the output of a radiation detector is directly dependant upon the total flux that is incident upon the sensitive area of the detector, which will be assumed in this chapter to be the area of the input aperture. Therefore, the 
basic equation relating the two detector signals and the radiation from the sources is simply:

$$
\frac{i^{\mathrm{T}}}{i^{\mathrm{S}}}=\frac{\Phi^{\mathrm{T}}}{\Phi^{\mathrm{S}}}
$$

The geometric resolution components placed before the detector, such as shown in Figure 13, are designed to ensure that only the desired flux is input to the detector. In the case of intensity and irradiance/illuminance measurements, the geometrical factors are the distance $d$ and the aperture area A. Although the intensity of a (point) source does not change with distance, the flux $\Phi$ that is incident upon a detector with a fixed input aperture A varies with the square of the distance as we discussed in Section 4.1.7.1. Therefore any comparison of two intensity sources (or irradiance/illuminance sources) will depend upon the distance at which we measure their output using a detector. If we allow the distances of the test and standard intensity sources from the detector aperture to be different, $d^{\mathrm{T}}$ and $d^{\mathrm{S}}$ respectively, the detector signal ratio is given by:

$$
\frac{i^{\mathrm{T}}}{i^{\mathrm{S}}}=\frac{\Phi^{\mathrm{T}}}{\Phi^{\mathrm{S}}}=\frac{I^{\mathrm{T}} \cdot A}{\left(d^{\mathrm{T}}\right)^{2}} \cdot \frac{\left(d^{\mathrm{S}}\right)^{2}}{I^{\mathrm{S}} \cdot A}=\frac{I^{\mathrm{T}}}{I^{\mathrm{S}}} \cdot\left(\frac{d^{\mathrm{S}}}{d^{\mathrm{T}}}\right)^{2}
$$

from which we obtain the test source intensity as

$$
I^{\mathrm{T}}=I^{\mathrm{S}} \cdot\left(\frac{d^{\mathrm{T}}}{d^{\mathrm{S}}}\right)^{2} \cdot \frac{i^{\mathrm{T}}}{i^{\mathrm{S}}}
$$

To reduce errors and uncertainties, we usually try to set up our measurements such that $d^{\mathrm{T}} \approx d^{\mathrm{S}}$ and that this distance is large enough that the inverse square law is valid.

If we combine Equations (8) and (9) together with Figure 12, we see that an intensity source may be used to calibrate an irradiance/illuminance meter:

$$
E^{S}=\frac{\Phi^{S}}{A}=\frac{I^{S}}{\left(d^{S}\right)^{2}}
$$

The input aperture area of the detector has cancelled out of the equations.

\subsection{Radiance/luminance}

A very convenient method used to produce a standard radiance or luminance source uses an isotropic diffusing reflecting surface to produce a known radiance from a known irradiance incident upon the surface, as discussed in Section 4.1.7.3 above. A schematic for the measurements is given in Figure 14. Note that the known irradiance at the diffuser may be produced by either a standard radiant intensity source (Equation (38)) or a standard irradiance source. For luminance, the known sources may also be either a standard luminous intensity source or a standard illuminance source. 


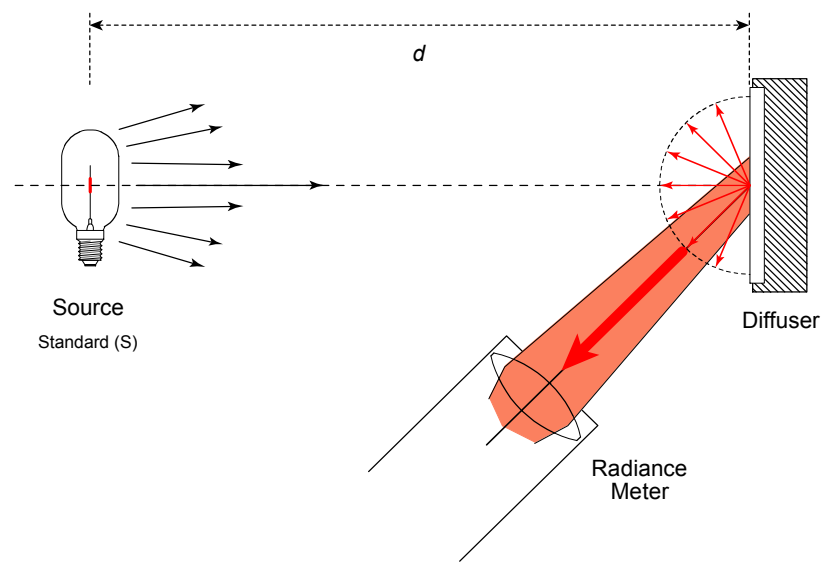

Fig. 14. Radiance source derived from an irradiance source with diffuser

\subsection{Optical radiation measurement standard sources}

The standard radiation sources discussed in the above examples are generally incandescent lamps. Since their output cannot be calculated directly from the properties of the lamps, these sources must be calibrated from primary measurement standards traceable to the SI as indicated in Section 3 above and discussed in Section 7 below. However, with care, incandescent lamps are excellent secondary, reference and working measurement standards that can be used to transfer calibrations from the primary standards realised in NMIs to the working standards used in many calibration laboratories. The choice and use of these lamps as standards requires consideration of their spectral, geometrical, electrical, mechanical and recalibration characteristics (CIE 149:2002).

\subsubsection{Spectral characteristics}

The output spectral distributions of incandescent lamps are particularly suitable for use as standards because the spectral distribution is a smooth and continuous function of the wavelength such as shown in Figure 8. This reduces the errors and uncertainties associated with measurements of their spectral distributions in narrow wavelength bandwidths. There are three main types of incandescent lamps characterized by the different gas fillings. Bulbs with no filling, or vacuum bulbs, can be used up to a maximum CCT of approximately 2400 $\mathrm{K}$ before the effects of filament evaporation become unacceptable. General lighting service bulbs filled with nitrogen and an inert gas at approximately atmospheric pressure may be used from approximately $2000 \mathrm{~K}$ to $2900 \mathrm{~K}$ CCT before the evaporation becomes excessive. Tungsten-halogen lamps, which operate with a gas filling pressure between 7 to 10 times atmospheric pressure and with a hot quartz envelope, may be used up to CCTs of approximately $3400 \mathrm{~K}$.

\subsubsection{Geometrical characteristics}

The construction of the bulb and the filament characteristics of these lamps can be somewhat adjusted to facilitate their application to the different geometrical quantities discussed. 
The lamps used for total flux in integrating spheres are usually designed with spherical bulbs and circular or distributed filament shapes to provide a uniform spatial output for a uniform illumination of the sphere walls.

The lamps used for intensity and illuminance are designed with planar filaments that enables the distance between the lamp and detector to be determined reproducibly and accurately. The bulb of the lamp is also shaped, such as a triangular shape, to reduce the inter-reflections inside the bulb that will cause scattered light errors in the measured output of the lamp. Spectral irradiance lamps are usually of the tungsten-halogen design operating with a CCT of approximately $3200 \mathrm{~K}$ to increase the amount of UV radiation. The deviation of the irradiance from these lamps from the inverse-square-law should be measured if they are to be used at different distances from that at which they were calibrated.

Sources used for luminance and radiance standards are often the irradiance/diffuser combination described in Section 5.3. If a higher radiance is required, ribbon or stripfilament lamps can be used. Present lamps of this type are limited to approximately $2900 \mathrm{~K}$.

\subsubsection{Electrical characteristics}

The filament of the incandescent lamp is heated by passing an electrical current through it. For measurement standard lamps, this is usually a direct current. The same polarity of the electrical current must be used each time the lamp is operated. To avoid thermal shock to the filament, this current should be applied gradually, over times on the order of a minute or more. When the lamp is calibrated, the current or voltage is adjusted until the spectral radiant output of the lamp reaches the desired operating CCT. This electrical operating point, either a defined operating current or voltage, must be applied whenever the lamp is used to obtain the same radiant output each time the lamp is used. Since all incandescent lamps will age with use, the second of the two electrical quantities (voltage, if the lamp current is the defined operating variable) may be used as a monitor of the ageing of the filament and consequent degradation of the lamp output calibration.

The electrical quantities must be measured accurately since the radiant output of an incandescent lamp depends strongly upon the electrical power applied to the lamp. Since the electrical power causes a change in temperature, the spectral distribution of the radiant output changes as well as the absolute amount of output. For lamps operating with a CCT of approximately $2856 \mathrm{~K}$, it has been observed (CIE 149:2002) that the luminous output of the lamp changes approximately $4 \%$ for a $1 \%$ change in lamp operating voltage and approximately $8 \%$ for a $1 \%$ change in lamp operating current.

\subsubsection{Mechanical characteristics}

Incandescent lamps are sensitive to vibration and shock. In addition to breakage of the glass envelope or the electrical feed-throughs, the filament structure is particularly fragile. As indicated above, electrical power should be applied gradually to avoid thermal shock.

The electrical and mechanical properties of a lamp change rapidly when a lamp is first used. Before a lamp is calibrated, it is usually aged by operating at the desired CCT for a period of time to enable the components of the lamp to stabilise, and to determine when the aging rate has stabilised. If measurements are made of the lamp output over time at a constant 
electrical operating current or voltage, the data may be used to estimate the change in the lamp calibration with later use.

\subsubsection{Recalibration}

Every lamp is an individual. Although an estimated aging rate may be determined as indicated above, the lamp may change suddenly for no apparent reason. The best method to monitor the behaviour of a single lamp is to keep records of every use and to check for any change in the electrical behaviour of the lamp. In a laboratory where accuracy is paramount, measurements should be made with more than one standard to ensure agreement between measurements. When the difference between standards becomes larger than acceptable, consideration must be given to a recalibration of the standards. If the lamps have been used extensively and are changing rapidly, it may be necessary to discard the lamp. However, since standard lamps are costly, sometimes difficult to obtain, and the calibration process is costly, a recalibration of existing lamps should be a first consideration.

If the standard lamps are extensively used in the measurement laboratory, consideration should be given to using the purchased calibrated sources as reference measurement standards to calibrate a set of working measurement standards that can be calibrated in the measurement laboratory and used for internal calibrations.

\section{Spectroradiometry}

One of the basic characteristics of a radiant source is the spectral distribution of the radiant output. As we have seen, even the accurate determination of photometric and colorimetric quantities requires knowledge of the spectral distribution of the radiation. The spectral distribution of the radiation in each of the geometrical quantities that we have discussed may be determined. Since the spectral techniques will be similar in each case, we will illustrate the methods using spectral irradiance as the example. In addition, since spectral irradiance can be used for a large majority of the calibration requirements, it is the most commonly available facility in a laboratory. Several references available for more information concerning spectroradiometric measurements are (CIE 063-1984, Grum \& Becherer, 1979, Kostkowski, 1997).

The basic instrument for spectral measurements is a spectroradiometer, which is an instrument for measuring radiometric quantities in narrow wavelength intervals over a given spectral region (CIE S017, 2011). An example of a spectroradiometer measurement configuration for spectral irradiance measurements is shown in Figure 15. The monochromator shown is a grating instrument with a prism predispersor input to remove the high-order wavelengths that would be passed by the grating components. There are two detector output ports with manual switching that allows several wavelength regions to be measured during one measurement set. Detectors such as photomultiplier tubes (PMT), and $\mathrm{Si}, \mathrm{Ge}$, InGaAs and InSb photodiodes are available to allow measurements covering the wavelength range from $200 \mathrm{~nm}$ to $2500 \mathrm{~nm}$. Various manually interchangeable gratings with different dispersions and blaze are available for use in measuring the same wavelength range. The purpose of the conical radiation trap is to remove radiation emitted in the back direction from scattering back into the input. The aperture defines the spatial region at the spectral irradiance source from which radiation is accepted at the input to the integrating sphere. 


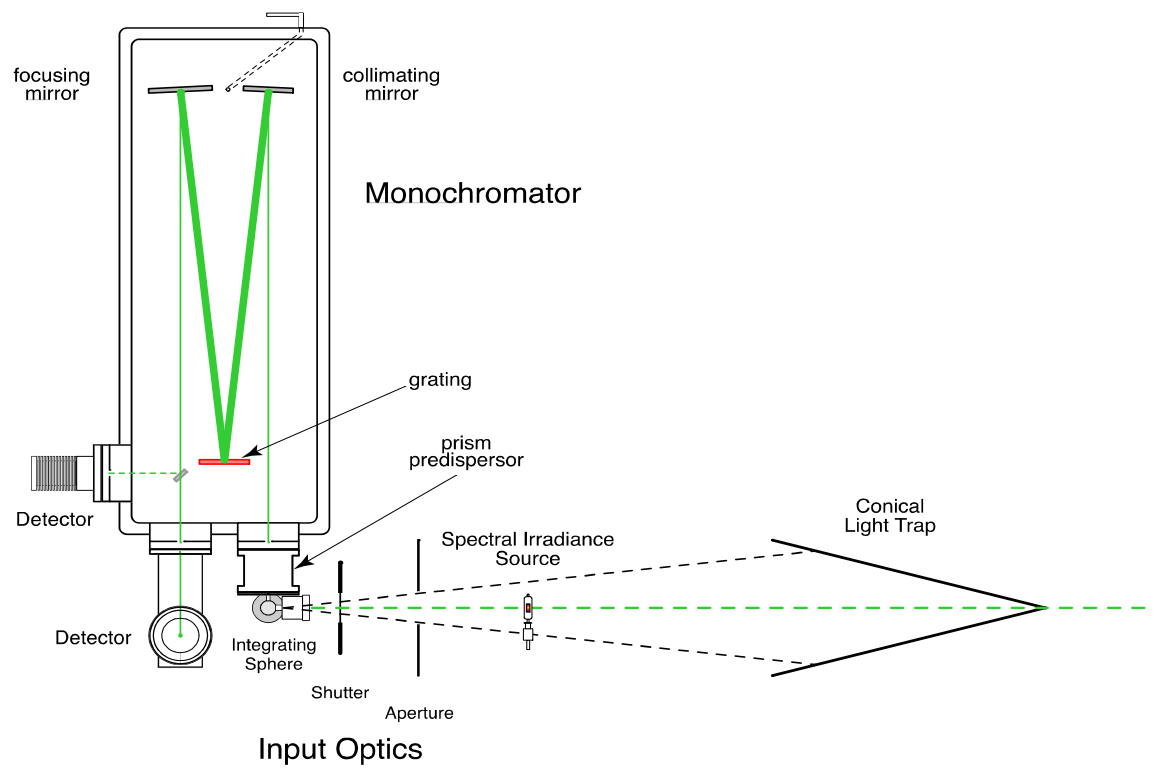

Fig. 15. Spectroradiometer and spectral irradiance measurement

A spectroradiometric measurement system may be considered to be composed of seven basic components (Figure 16): i) the radiation source, ii) the input optics that couples the desired radiation into the monochromator, iii) the monochromator, iv) the output optics that couples the radiation from the monochromator into the detectors, v) the detector, vi) a measurement control system, and vii) some signal processing equipment.

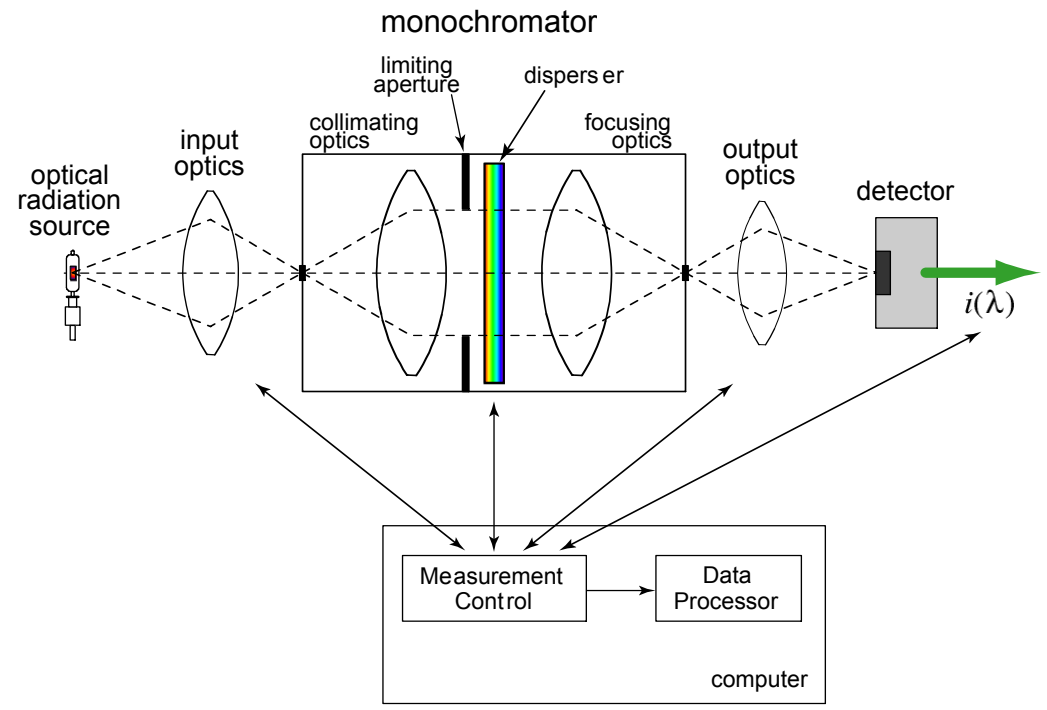

Fig. 16. General configuration for spectroradiometric measurements 
For simplicity, in Figure 16 and other figures, lenses (refractive elements) are used to show the focusing of radiation. In most cases, mirrors (reflective elements) are preferable since they will focus all wavelengths of radiation to the same physical spot, whereas lenses do not. For example, the monochromator in Figure 15 uses mirrors in the collimating and focusing optics.

\subsection{Monochromator}

The basic components of a monochromator are shown in Figure 16. The disperser may be a grating or a prism. The limiting aperture serves to define the size of the radiation beam that passes through the monochromator, and limits the size and shape of the input beam that passes through the monochromator. The collimating optics transforms the diverging input beam from the input slit into a parallel beam of radiation incident upon the disperser. The focusing optics focuses the dispersed beam from the disperser onto the output plane at the position of the output slits. The optical and wavelength dispersion properties of a monochromator are best understood by noting that there are two kinds of optics in a monochromator. One kind forms images of the source (input slit) at the output plane (output slit), as shown by the dashed lines inside the monochromator of Figure 16. The optical magnification of the system is usually one, so that the image of the input slits produced at the output plane is the same size as the input slits. The second kind of optics disperses the radiation. For gratings and prisms, this dispersion is spatial with the beams of different wavelengths effectively propagating into different directions from the disperser. As a result, there are many images of the input slits produced at the output plane, one for each of the wavelengths present in the input radiation. Due to the finite width of the input slit and the image, and the continuous wavelength range of most radiation sources, the images of different adjacent wavelengths will partially overlap and there will be a range of wavelengths that are passed through the output slit. This effect gives rise to the bandwidth property of a monochromator.

\subsection{Spectroradiometer input optics}

The purpose of the spectroradiometer input optics is to couple the desired radiation flux from the source into the monochromator as efficiently and accurately as possible. The type of coupling used should take into account the characteristics of the complete system that influence the accuracy of our measurements. Some issues to consider are:

i. Any non-uniformity in the transmission of any of the optical components, or in the spatial responsivity of the detector, will cause an incorrect 'weighting' of different parts of the spatial radiation distribution. This non-uniformity could depend both upon the direction of the incident radiation, as well as the position of the input radiation. To reduce this effect, the radiation that is input to the monochromator is usually made as spatially uniform as possible.

ii. Some of the system components will be affected by the polarization of the radiation. This is particularly important when using grating monochromators. The responsivities of some detectors are also affected by the polarization of the incident radiation. This effect will cause an incorrect 'weighting' of different polarization components of the radiation beam. These issues can be resolved by measuring the radiation separately for each polarization, or by spatially mixing the input radiation to provide a uniformly polarized beam. 
iii. Imperfections in the optical components will cause scattering of some of the radiation from its ideal trajectory. This causes both a loss in signal and stray radiation problems.

iv. The efficiency of our optical design and components will affect our signal-to-noise and accuracy. Since the signals we are usually measuring with a spectroradiometer are small, one of the prime considerations is to collect as much radiation as possible, and to use it as efficiently as possible, without undue loss anywhere in the optical path. To this end, we should use as much of the optical system, particularly that of the monochromator, as possible. The basic ideas of filling the input optics of the monochromator are shown in Figure 17.
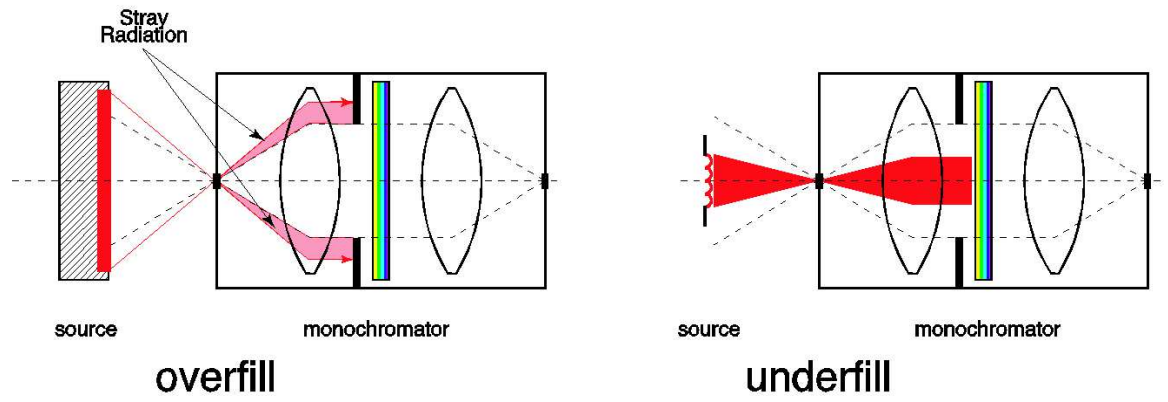

Fig. 17. Source size and monochromator optics

If the radiation from the source overfills the monochromator optics, the overfill radiation will become stray scattered radiation within the monochromator. The problem with this stray radiation is that radiation of wavelengths other than those we are measuring can enter our detection system and cause an erroneous addition to our output signal. A small source, or a source placed at a large distance from the monochromator, will underfill the monochromator optics. In addition to being inefficient, this underfilling of the optics is susceptible to any non-uniformity in the optics. Any motion of the source will cause the radiation to pass through a different part of the spectroradiometer optics, which may result in a different response from the monochromator. This will become more of a problem if another source is used to calibrate the spectroradiometer. This other source will probably be a different size and therefore pass through different optical paths in the system.

v. An input optic lens or mirror system that collects more radiation from the source and is sized to fit the monochromator input is often used. The schematic of Figure 16 shows this configuration. In this case the filament of the lamp is focused onto the input slits of the monochromator. There are two problems with this arrangement: 1.) Since an image of the filament is produced on the slits, any vibration of the radiation source or spectroradiometer components will cause a dramatic variation in the amount of radiation that passes through the slits and enters the monochromator. 2.) The spatial structure of the radiation at the input slits will be imaged onto the output slits of the monochromator, and then into the detector. If there is any non-uniformity in the detector spatial responsivity, our output signal will not be representative of the amount of input radiation.

There are, however, instances when we do use this type of input optics. These are usually when the source is a uniform source, such as a Lambertian diffuser, or when we 
are imaging the radiation emitted from a certain portion of a ribbon filament of an incandescent lamp. In both of these instances, the source has very little spatial structure.

vi. In many radiometric measurements, the input system involves a diffuser, either a flat plate (Figure 17) or an integrating sphere. While this may cause a considerable loss of radiation, it does reduce the errors caused by geometrical structure in the radiation source, component vibration, polarization effects, and any spatial non-uniformity in the monochromator optics and/or detector responsivity. The input optics to the monochromator shown in Figure 15 is an integrating sphere, together with a baffle assembly. More detail for this configuration is shown in Figure 18.

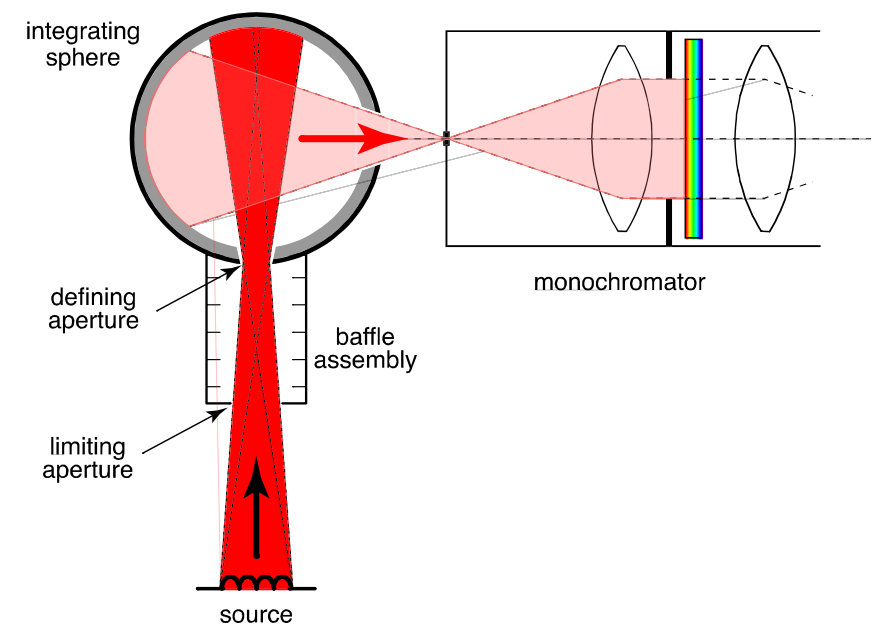

Fig. 18. Integrating sphere input to monochromator

The input port to the sphere is the area that defines what flux is to be measured, so it acts as part of the defining optics for the radiation source measurement. The required distance is that between the source and the defining aperture at the sphere input. The size of the sphere and its ports should be arranged such that the radiation that enters the monochromator has had more than one 'bounce' within the sphere. Figure 18 shows a configuration such that the region of the sphere wall that emits radiation into the monochromator does not overlap with the region of the sphere wall that is directly irradiated by the source. Integrating spheres accept radiation from all directions and emit radiation into all directions at any open port of the sphere. This must be controlled to avoid stray radiation from entering the sphere and to prevent an overfill of the input optics to the monochromator as was shown in Figure 17. To prevent on overfill at the monochromator, the size of the sphere output port and the distance of the sphere output port from the monochromator input slits can be adjusted such that the optics of the input to the monochromator are just filled, as shown in Figure 18.

A baffle assembly may be used at the input to the integrating sphere to reduce unwanted stray radiation from entering the sphere. Apertures and baffles must be considered carefully, since the edges of the aperture or baffle, no matter how thin, will reflect significant radiation, especially from the direct input beam, into the measured portion of the input. The baffle assembly shown in Figure 18 includes only one baffle, the limiting front aperture, that 
directly views the input radiation from the source. The baffles interior to the assembly are only used to prevent scattered radiation from the interior walls of the baffle assembly from re-entering the input beam to the sphere. The limiting aperture is large enough that it does not define, or vignette, the final size of the input radiation beam that enters the sphere. Its purpose is to limit as much as possible the amount of off-angle stray radiation that enters the integrating sphere.

The multiple reflections at the interior of the sphere cause a change in the relative spectral distribution of the sphere by a factor of $\rho(\lambda) /(1-\rho(\lambda))$, where $\rho(\lambda)$ is the reflectance of the material of the sphere wall (CIE 084, 1989). Any irregularities or changes in $\rho(\lambda)$ with wavelength will become amplified in the sphere output radiation. With the typical white diffusers used in integrating spheres, such as PTFE or $\mathrm{BaSO}_{4}$, this is usually observed in the lower wavelengths below approximately $400 \mathrm{~nm}$. If a more uniform behaviour with wavelength is desired, a compromise is to reduce the reflectance $\rho(\lambda)$ from its high values near $99 \%$ to approximately $85 \%$ by addition of dark absorbers into the coating.

In summary, although the integrating sphere input configuration has several disadvantages, the advantages that it provides in reducing the spatial non-uniformities of sources and detectors outweigh the disadvantages. The disadvantages may be mitigated by careful consideration of each of the components of the system when assembling a measurement system.

\subsection{Spectroradiometer output optics and detector}

The purpose of the output optics is to couple the dispersed radiation from the monochromator, at the monochromator output slit, onto the detector. To obtain the maximum signal-to-noise at the detector, the detector is often placed as close to the output slit as possible in order to collect all the radiation. If the detector must be placed at some distance from the output slits of the monochromator, some optics will be needed to collect the radiation and re-image it onto the detector. Note that if the beam is focused too intensely onto the detector, local overloading of the conversion process from photons to the output (electrical) signal may occur, causing non-linearity in the detector response.

The spectroradiometer shown in Figure 15 is designed to enable various detectors to be used depending on the wavelength range of interest. Each of these detectors requires different output optics of the spectroradiometer to couple the radiation from the monochromator to the sensitive area of the detector. For the VIS and NIR wavelengths, a silicon (Si) photodiode and a InGaAs photodiode can be mounted directly behind the slits. For the UV and VIS wavelengths, a photomultiplier (PMT) is used with a lens assembly to focus the radiation from the slits onto the PMT cathode. The assembly for the infrared (IR) detectors (Ge and $\mathrm{InSb}$ ) is a bit more complex since these detectors are often cooled with liquid nitrogen and prefer to be operated in an upright position. In addition, their sensitive areas are usually quite small. An elliptical mirror may be used to change the direction of the radiation beam, to focus the radiation onto the detector, and to avoid the problem of a change in the focal position with wavelength (Gaertner \& Boivin, 1995).

\subsection{Measurement procedures}

The basic concepts of a measurement and the associated calibration procedures are the same for spectroradiometric measurements as presented in Section 5 above. A measurement is a 
comparison between our unknown spectral quantity and a similar quantity of known magnitude. The comparison device or transfer device is now a spectroradiometer, which is a little more complex than the single detectors or photometers we discussed. The input optics to the spectroradiometer must define the geometric quantity that we wish to measure, but the spectroradiometer itself only puts out a signal, such as the current from the detector, which is related to the radiant flux at the input to the spectroradiometer. The basic measurement Equation (34) now becomes a function of wavelength, to be applied at each wavelength that is measured.

$$
S^{\mathrm{T}}(\lambda)=\frac{i^{\mathrm{T}}(\lambda)}{i^{\mathrm{S}}(\lambda)} \cdot S^{\mathrm{S}}(\lambda) \cdot \frac{T^{\mathrm{S}}(\lambda)}{T^{\mathrm{T}}(\lambda)}
$$

The term $T(\lambda)$ indicates the effect of all the components of the spectroradiometer, from the input optics to the detector (Figure 16), upon the input source flux $S(\lambda)$. Usually we assume that the effect of $T(\lambda)$ upon the measurements is the same for both the test and the standard sources, which reduces Equation (39) to the familiar form of Equation (34) for each wavelength. In spectroradiometric measurements the terms that must remain constant between the measurement of the standard and the test quantity are quite complex. Characteristics of the monochromator such as the large peaks in the grating reflectance and the reproducibility of the wavelength scale place greater demands on the ability of the instrument to reproduce its settings. The effects of these two particular characteristics can be mitigated by performing the measurements on both sources at each wavelength, before adjusting the monochromator to the next required wavelength. This requires that switching the monochromator between the test source and the standard source can be done in an accurate and reproducible manner. In addition to requiring the reproducibility of the monochromator, the entire optical system must 'evaluate' the flux in the same manner for the two measurements. This means that nothing in the behaviour of $T(\lambda)$ should depend on the magnitude of $S(\lambda)$, nor its initial direction or position within the defined path.

\section{Primary radiation sources and calibration chains}

In Section 3 we introduced the concept of a metrological traceability chain that enables our measurements to be accurate and reproducible worldwide. The primary measurement standards used for optical radiation measurements may be traceable to either detector standards or source standards that provide a procedure by which the reference quantity is the definition of the measurement unit through its practical measurement. The two sources that are typically used in the source-based method are (1) blackbody radiators, whose output is calculated from the Planck equation when the temperature of the blackbody is known (Mielenz et al, 1990), and (2) synchrotron radiators, whose output can be determined from the calculable radiance of accelerating charged particles (Ulm, 2003). The detectorbased method depends upon the measurement of absolute quantities of radiant flux by absolute radiometers (Boivin \& Gibb, 1995), which compare the heating caused by the absorption of the radiant flux with the heating caused by a known amount of electrical power. A comparison of the basic optical radiation calibration chain (Gaertner, 2009) for each of these methods, using a high temperature blackbody radiator source (HTBB), is given in Figure 19. 


\section{DETECTOR-BASED SOURCE-BASED}

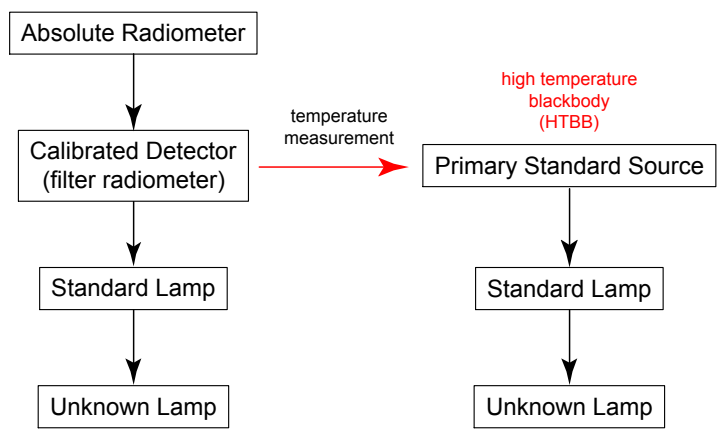

Fig. 19. Detector-based and HTBB Source-based calibration chains for optical radiation measurements.

This calibration chain indicates that HTBB primary source based calibrations are based upon primary detector measurements. The absolute temperature of a HTBB must be determined by the absolute measurement of the radiance of the HTBB. This is done using filter radiometers that measure the radiance or irradiance of the HTBB within a limited wavelength range. The filter radiometers used for irradiance measurements (Boivin et al, 2010) are basically constructed from detectors with a wavelength-limiting filter that allows the spectral responsivity of the filter radiometer to be accurately calibrated from measurements traceable to the absolute cryogenic radiometer. The measurement of the radiance of the HTBB is performed using the geometry described in Section 4.1.7.4, with a calibrated filter radiometer as the receiver. After the temperature of the HTBB is determined, the spectral radiance of the HTBB may be calculated for the desired wavelength range using the Planck equation. The HTBB may then be used for spectral irradiance calibrations using the geometry described in Section 4.1.7.4. With HTBB operating temperatures from $2500 \mathrm{~K}$ to $3500 \mathrm{~K}$, known spectral irradiances may be produced from wavelengths of $200 \mathrm{~nm}$ to over $2500 \mathrm{~nm}$ to calibrate standard incandescent lamps, such as the $1000 \mathrm{~W}$ FEL lamps that are commonly used as spectral irradiance measurement standards. Since the radiation output of an incandescent lamp is quite low in the UV, deuterium arc lamps are often used in the wavelength range from $200 \mathrm{~nm}$ to $350 \mathrm{~nm}$. These can be calibrated using HTBB sources operating in the upper range $(3400 \mathrm{~K}$ to $3500 \mathrm{~K})$ of their temperature limits.

Whereas the transfer from detector-based to source-based measurements is very useful for spectral radiation measurements, the calibration of sources that are used for photometric measurements is best performed using filter radiometers directly to calibrate the standard incandescent lamps (Gaertner et al, 2008). For this purpose, the filter radiometer is constructed with a combination of filters that results in a filter radiometer with a spectral responsivity that approaches the photometric functions as closely as possible.

\section{Uncertainties}

A measurement is not complete until an estimate of the uncertainties has been made and an uncertainty budget prepared. The GUM (JCGM, 2008a) and related documents (JCGM, 2009) 
are excellent references. In addition, the CIE has published an extensive document on uncertainty determinations (CIE 198:2011).

The measurement configurations discussed in the preceding sections have served to define the typical types of optical radiation measurements. This means that we will be measuring radiation using internationally defined quantities and units. This requires that the actual measurement configurations and equipment that we use must adhere to the internationally accepted definitions.

There are many factors that will influence the results of our measurements. In addition to the difficulty of configuring our apparatus to measure the quantities we wish to measure, there will be influences of time, temperature, humidity and many other often unknown or unexpected factors upon the equipment we use to give us our results. The consequences are that the results we obtain are not exactly what we wish to obtain, nor are they what we would like to claim them to be. Our only recourse is to try to understand the behaviour of our equipment and how many of the conditions in our laboratory can influence the results. In many cases this will mean a deliberate attempt to change these variables to determine the subsequent change in our results. By this means we will come to know the influences that change the results by significant amounts, and are therefore important influences to control. If we cannot control them, we will at least have an idea as to how to correct our results to account for the error caused by them, or at minimum, the amount of an uncertainty to apply to our results.

The evaluation of measurement uncertainties has been divided into two different types: Type A and Type B (JCGM 100:2008, JCGM 104:2009, JCGM 200:2008).

\subsection{Type A uncertainty evaluation}

The Type A evaluation of a component of our measurement uncertainties is done by a statistical analysis of the measurement quantity values that are obtained by repeated measurements under the same defined measurement conditions. Each measurement value under these conditions is different from the previous measured value in a random manner such that the next measured value cannot be predicted exactly from the previous value. These uncertainties are usually analysed using Gaussian probability density functions, such as shown in Figure 20. If many repeated measurements are made, the distribution describing the quantity $x$ will approach a curve similar to the black curve labeled 'distribution of data'. From these measured values, $x_{\text {mean }}$, the mean value of $\mathrm{x}$, and $\sigma_{\text {data }}$, the standard deviation of the distribution of the measured data, can be calculated. Any single measured value of $x$ is expected to be found within this distribution.

The accuracy or uncertainty with which we know both $x_{\text {mean }}$ and $\sigma_{\text {data }}$ depends upon how well we know the 'distribution of data' curve, which will depend upon how many data points we have taken. The accuracy of $x_{\text {mean }}$ is described by the standard deviation of the mean, $\sigma_{\text {mean }}$, which is calculated from the datapoints by:

$$
\sigma_{\text {mean }}=\sigma_{\text {data }} / \sqrt{n}
$$

where $n$ is the number of datapoints. The red curve labeled 'distribution of mean' in Figure 20 shows the uncertainty with which the mean value $x_{\text {mean }}$ is known from $n$ measurements 
of $x$, all of which are assumed to be part of the distribution shown by the distribution of data' curve, and as calculated for a Gaussian distribution using equation (40). The value used for $n$ was 10 in Figure 20.

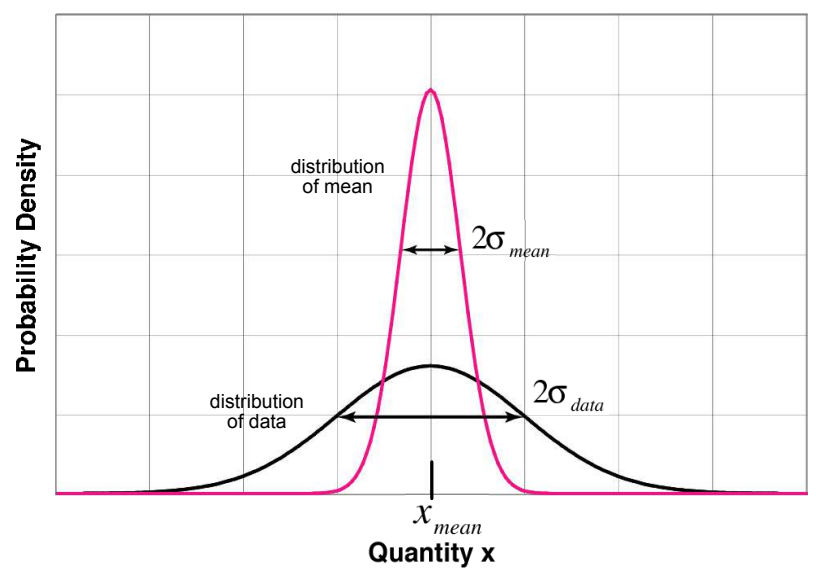

Fig. 20. Gaussian probability density functions for Type A evaluation of uncertainties.

The distinction between $\sigma_{\text {data }}$ and $\sigma_{\text {mean }}$ is important for the estimation of the Type A uncertainties in the calibration and use of optical radiation detectors, such as photometers and spectroradiometers. During the calibration of the detector, a total of $\mathrm{n}$ measurements may be performed, resulting in a mean calibration value of $x_{\text {mean }}$ with a Type A uncertainty of $\sigma_{\text {mean }}$. When the detector is later used for a single measurement of quantity $\mathrm{x}$, using the calibrated value obtained from $x_{\text {mean }}$, the Type A uncertainty in this single measured value will be given by $\sigma_{\text {data }}$, the standard distribution of the probability distribution that describes the repeatability of the detector. The uncertainty in the calibration of the detector, which includes the $\sigma_{\text {mean }}$ obtained during the calibration, now becomes an uncertainty with a Type $B$ uncertainty evaluation for the single subsequent measurement.

\subsection{Type B uncertainty evaluation}

The Type B uncertainty evaluation is defined as an evaluation of a component of measurement uncertainty determined by means other than a Type A evaluation uncertainty (JCGM, 2008b). This type of evaluation is used with systematic errors, which are themselves associated with the fact that a measured quantity value contains an offset from the true quantity value. These offsets have many origins, such as those indicated in Section 8.0 above. Determining actual and potential systematic errors requires critical evaluation of our measurement configuration.

As we have seen, optical radiation measurements involve the determination of the flux produced by a source into many different geometrical configurations. As a result, these measurements will require the use of working standard sources to either calibrate our detection system or to compare with our test source using the detection system. We noted in Section 4.2.5 the importance of comparing sources as similar as possible to reduce the spectral errors and uncertainties in photometric measurements. In Section 6.4 we discussed 
the importance of accounting for the potential differing responses of the spectroradiometer $(T(\lambda))$ to the different sources. As may be expected, this 'like-with-like' principle is also applicable to geometrical properties and in signal size comparisons. Therefore we should use a working standard lamp that is as similar in relative spectral output, geometrical size and shape, and flux output as the test lamp we wish to measure. By using all these techniques we will stack as many odds in our favor as possible.

However, as discussed below, there will still be aspects of our measurement that will cause us some errors and uncertainties.

\subsubsection{Radiation sources}

Some of the properties of optical radiation measurement standard sources were introduced in Section 5.4. The following sources of error and uncertainty must be considered for both the working standard and the test sources:

1. The calibrated value from the Calibration Laboratory will indicate an uncertainty in the value that has been placed on the working standard source. This is your minimum uncertainty, since you cannot do anything about it.

2. The source calibration is given for defined electrical operating conditions. The uncertainty with which you can reproduce the defined current (or voltage) through the lamp will cause an uncertainty in the amount of radiation that you are receiving from the lamp. As indicated in Section 5.4.3 above, this can be a large effect. This uncertainty will be dependent upon the calibration of the voltmeter and the standard resistor that are used to set the lamp current. The ability of the power supply to fine-tune the electrical values to obtain the defined values will affect the lamp output. In addition, the short-term fluctuations in the electrical output of the power supply will add to instability in the source radiation.

3. The calibration value is true for a defined geometric alignment of the source. This usually means the reference point in the lamp filament and the direction from the plane of the lamp filament for which the calibration was made. The source output may be different in different directions from the filament. If this information is known, or if you can measure it, an uncertainty may be calculated due to your uncertainty in exactly reproducing the original calibration conditions.

4. The intensity of the source may vary with distance from the lamp, or the irradiance due to the source may not follow the inverse-square-law. To test this for your measurement configuration, and for accurate results, a series of measurements at different distances from the source should be performed to test the inverse-square-law behaviour of the lamp (see Section 4.1.7.1).

5. The irradiance from the source at the detector may not be spatially uniform. Every measurement is actually an average irradiance over the full area of the acceptance aperture of the detector. Any difference in the size of the spatial region of the source irradiance field between that calibrated by the Calibration Laboratory and that used in your measurements will result in an error due to this spatial non-uniformity.

6. The radiation output of incandescent lamps changes with use. This aging effect can be approximated to enable a correction to be made, but there will be an associated uncertainty with this correction. This aging effect for each lamp may be approximated from measurement data that can be made when the lamp is originally aged before calibration (Section 5.4.4). 


\subsubsection{Radiation detectors}

The complexity of photometers and spectroradiometers gives rise to many interesting sources of error.

1. The failure of a photometer to match the desired photometric or colorimetric functions has been discussed in Section 4.2.5. The uncertainty in the spectral mismatch correction factor will need to be determined from the estimated uncertainties in the spectral distribution functions for the contributing sources and the photometer.

2. The response of the detector used in the photometer or spectroradiometer to input flux signal size must be linear to allow comparison of sources with differing amounts of radiation output. This effect can also include the associated electronics.

3. If the geometric distribution of the output of the lamps varies with angle or position, and if measurements of high accuracy are required, any spatial non-uniformity in the responsivity of a photometer will have an effect. The use of integrating spheres to mitigate this source of error was discussed in Section 6.2.

4. If the number of digits available in the output of the voltmeter used to measure the signal size is small, the measured signals will be subject to a digitizing error.

5. Monochromator wavelength errors. There are two possible components to this error: the deviation of the indicated monochromator wavelength from its actual value, and the ability of the monochromator to reproduce this error. The reproducibility error may give rise to a random error that can be determined using a Type A uncertainty evaluation if enough repeatable measurements can be made. The effects of an error in the monochromator wavelength calibration will predominantly depend upon the difference in the relative spectral distribution of the two sources that are being compared. If the sources have the same relative spectral distribution, the wavelength offset will cause the same relative effect for both sources, resulting in no error in calibration.

6. Spectral bandwidth errors. The signal at the spectroradiometer detector is a weighted average over the spectral bandwidth of the monochromator. If the spectral distribution of the source changes rapidly over this bandwidth, much of this spectral information will be lost in the averaging process.

More detail concerning these and other effects may be found in several of the references (CIE 063:1984, Grum \& Becherer, 1979, Kostkowski, 1997).

\section{Conclusion}

The measurement of absolute amounts of optical radiation requires careful and detailed consideration of a broad range of physical concepts and practical instrumentation to produce an accurate, reproducible and internationally acceptable result. The basis for this is the internationally defined terminology and definitions of the measurement quantities and accepted units that are useful for optical radiation measurements. We have discussed the five predominant measurement quantities and indicated the measurement configurations necessary to obtain reliable results. The geometrical relationships between these quantities have been discussed with the purpose of allowing measurement standards to be used for several different measurement quantities. Some of the requirements to measure optical radiation in a means that is relevant to the human visual response to radiation have been presented. The characteristics of incandescent sources that are a basis for most measurements have been discussed, together with the sources of errors and uncertainties that these 
characteristics present. The basic concepts of the photometers and spectroradiometers that are used to measure the radiation output from these sources have been presented with the aim of providing a reliable foundation for basic metrology and applied measurements. The necessity of international acceptability of goods and services, energy efficiency, and consumer safety have encouraged the development of more accurate, versatile and reliable equipment both to produce a wider variety of radiation sources and better means of determining their suitability to the desired application. The basic concepts and techniques described in this chapter should provide the necessary tools to pursue these goals.

\section{References}

BIPM (2006), The International System of Units (SI), $8^{\text {th }}$ edition. Available from http://www.bipm.org .

Boivin, L.P. \& Gibb, K., (1995). Monochromator-based cryogenic radiometry at the NRC, Metrologia Vol. 32, No. 6, (1995), pp. 565-570.

Boivin, L.P., Bamber, C., Gaertner, A.A., Gerson, R.K., Woods, D.J. \& Woolliams. E.R. (2010) Wideband filter radiometers for blackbody temperature measurements, Journal of Modern Optics, Vol. 57, No. 17, (October 2010), pp. 1648-1660, ISSN 0950-0340.

CIE 015:2004 Colorimetry, 3rd Edition, ISBN 978-3-901906-33-6, Commission Internationale de L'Eclairage, Vienna, Austria.

CIE 063 (1984), The spectroradiometric measurement of light sources, ISBN 978-963-7251-238, Commission Internationale de L'Eclairage, Vienna, Austria.

CIE 069 (1987), Methods of Characterising Illuminance Meters and Luminance Meters, ISBN978-3-900734-04-6, Commission Internationale de L'Eclairage, Vienna, Austria.

CIE 084 (1989), The Measurement of Luminous Flux, ISBN-978-3-900734-21-3, Commission Internationale de L'Eclairage, Vienna, Austria.

CIE 127 (2007), Measurement of LEDS, ISBN 978-3-901-906-58-9, Commission Internationale de L'Eclairage, Vienna, Austria.

CIE 149 (2002), The Use of Tungsten Filament Lamps as Secondary Standard Sources, ISBN 3-901-906-18-5, Commission Internationale de L'Eclairage, Vienna, Austria.

CIE 179 (2007), Methods for Characterising Tristimulus Colorimeters for Measuring the Colour of Light, ISBN 978390190660 2, Commission Internationale de L'Eclairage, Vienna, Austria.

CIE 191:2010 Recommended System for Mesopic Photometry Based on Visual Performance, ISBN 978-3-901906-88-6, Commission Internationale de L'Eclairage, Vienna, Austria.

CIE 198:2011 Determination of Measurement Uncertainties in Photometry, ISBN 978-3902842-00-8, Commission Internationale de L'Eclairage, Vienna, Austria.

CIE Standard S 010/E:2004, Joint ISO/CIE Standard ISO 23539:2005(E) / CIE S010/E:2004 Photometry - The CIE System of Physical Photometry, Commission Internationale de L'Eclairage, Vienna, Austria.

CIE Standard S 014-2/E:2006, Joint ISO/CIE Standard ISO 11664-2:2007(E)/CIE S 0142/E:2006 CIE Colorimetry-Part 2: Standard Illuminants for Colorimetry, Commission Internationale de L'Eclairage, Vienna, Austria

CIE Standard S 017/E:2011, ILV: International Lighting Vocabulary, Commission Internationale de L'Eclairage, Vienna, Austria. 
Gaertner, A.A. \& Boivin, L.P., (1995). Some problems in realizing an infrared spectralirradiance scale from $1500 \mathrm{~nm}$ to $2400 \mathrm{~nm}$ at the NRC, Metrologia Vol. 32, No. 6, (1995), pp. 615-619.

Gaertner, A.A., Bamber, C., Boivin, L.P. and Chrysler, M. (2008) NRC photometer design for the realisation of a luminous intensity scale, CIE publication x033:2008 Proceedings of the CIE Expert Symposium on "Advances in Photometry and Colorimetry", 7-8 July 2008, Turin, Italy.

Gaertner, A.A., (2009). Spectral irradiance calibrations at the National Research Council of Canada (NRC), Journal of Modern Optics Vol. 56, No. 13, (July 2009), pp. 1488-1496, ISSN 0950-0340.

Gardner, J.L. (2000). Correlated Colour Temperature - Uncertainty and Estimation, Metrologia Vol. 37, No. 5 (2000), pp. 381-384.

Grum, F. and Becherer, R.J., (1979) Optical Radiation Measurements Volume 1 Radiometry, Academic Press ISBN 0-12-304901-6 (v.1), New York, USA.

Höpe, A. \& Hauer, K-O. (2010) Three-dimensional appearance characterization of diffuse standard reflection materials, Metrologia Vol. 47, No. 3, (June 2010), pp. 295-304.

JCGM 100:2008, Joint Committee for Guides in Metrology (September 2008), Evaluation of Measurement Data - Guide to the expression of uncertainty in measurement (GUM). Available from http://www.bipm.org .

JCGM 104:2009, Joint Committee for Guides in Metrology (July 2009), Evaluation of Measurement Data - An introduction to the "Guide to the expression of uncertainty in measurement" and related documents. Available from http://www.bipm.org .

JCGM 200:2008, Joint Committee for Guides in Metrology (2008), International vocabulary of metrology - Basic and general concepts and associated terms (VIM). Available from http:/ / www.bipm.org .

Kostkowski , H.J. (1997) Reliable Spectroradiometry, Spectroradiometry Consulting, ISBN-09657713-0-X, La Plata, Maryland, USA.

Mielenz, K.D., Saunders, R.D., Parr, A.C. \& Hsia, J.J. (1990), The 1990 NIST Scales of Thermal Radiometry, J. Res. Natl. Inst. Stand. Technol. Vol 95, No. 6 (December 1990), pp. 621-629.

Ohno, Y. (1997) Measurement Procedures (Chapter 5) and Photometric Standards (Chapter 3), In Handbook of Applied Photometry, Casimir DeCusatis, (133-177, 55-99) American Institute of Physics Press, ISBN 1-56396-416-3, Woodbury, NY, USA.

Ohta, N. \& Robertson, A.R. (2005) Colorimetry Fundamentals and Applications, Wiley, ISBN-13 978-0-470-09472-3, Chichester, England

Ulm, G., (2003). Radiometry with synchrotron radiation, Metrologia Vol. 40, No. 1, (February 2003), pp. S101-S106.

Walsh, John W.T. (1958), Photometry, 3rd Edition, Constable, London, UK. 


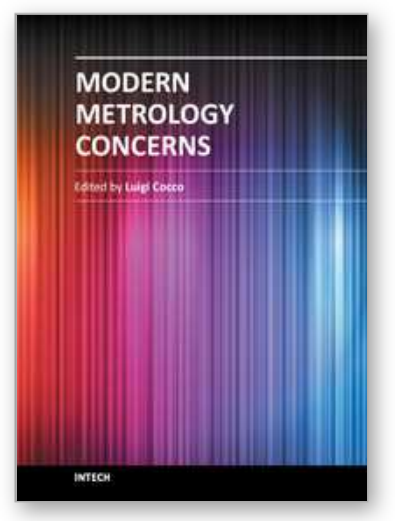

\author{
Modern Metrology Concerns \\ Edited by Dr. Luigi Cocco
}

ISBN 978-953-51-0584-8

Hard cover, 458 pages

Publisher InTech

Published online 16, May, 2012

Published in print edition May, 2012

"What are the recent developments in the field of Metrology?" International leading experts answer this question providing both state of the art presentation and a road map to the future of measurement science. The book is organized in six sections according to the areas of expertise, namely: Introduction; Length, Distance and Surface; Voltage, Current and Frequency; Optics; Time and Relativity; Biology and Medicine. Theoretical basis and applications are explained in accurate and comprehensive manner, providing a valuable reference to researchers and professionals.

\title{
How to reference
}

In order to correctly reference this scholarly work, feel free to copy and paste the following:

A. A. Gaertner (2012). Optical Radiation Measurement, Modern Metrology Concerns, Dr. Luigi Cocco (Ed.), ISBN: 978-953-51-0584-8, InTech, Available from: http://www.intechopen.com/books/modern-metrologyconcerns/optical-radiation-measurements

\section{INTECH}

open science | open minds

\author{
InTech Europe \\ University Campus STeP Ri \\ Slavka Krautzeka 83/A \\ 51000 Rijeka, Croatia \\ Phone: +385 (51) 770447 \\ Fax: +385 (51) 686166 \\ www.intechopen.com
}

\author{
InTech China \\ Unit 405, Office Block, Hotel Equatorial Shanghai \\ No.65, Yan An Road (West), Shanghai, 200040, China \\ 中国上海市延安西路65号上海国际贵都大饭店办公楼 405 单元 \\ Phone: +86-21-62489820 \\ Fax: $+86-21-62489821$
}


(C) 2012 The Author(s). Licensee IntechOpen. This is an open access article distributed under the terms of the Creative Commons Attribution 3.0 License, which permits unrestricted use, distribution, and reproduction in any medium, provided the original work is properly cited. 